Gazi University
Journal of Science
http://dergipark.gov.tr/gujs

\title{
An Adaptive Time Filter Based Finite Element Method for the Velocity- Vorticity-Temperature Model of the Incompressible Non-Isothermal Fluid Flows
}

\author{
Mine AKBAS* \\ Duzce University Faculty of Science and Letters, Department of Mathematics, 81620, Duzce, Turkey
}

Highlights

- This paper focuses on velocity-vorticity-temperature (VVT) model of the Boussinesq equations.

- A second order time accurate finite element method is proposed for solving these equations.

- Unconditional stability with respect to time step is proven.

\section{ArticleInfo \\ Received: $29 / 01 / 2020$ \\ Accepted: 10/03/2020}

\section{Keywords}

Finite element method

The Boussinesq equations

Time filter

\begin{abstract}
This paper studies a velocity-vorticity-temperature (VVT) model of the Boussinesq equations and introduces a numerical method for solving that. The proposed numerical method adds separate three minimally intrusive steps, one for each fluid variable, except pressure, to the standard semi-implicit backward-Euler (BE) approximation of VVTmodel. The key idea in these intrusive steps is to post-process the BE approximate solutions with 2-step, second order, linear time filters. The paper provides full mathematical analysis of the proposed numerical method, and two numerical experiments for that. The first numerical experiment verifies the predicted convergence rates while the second one shows the effectiveness of the method on a benchmark problem.
\end{abstract}

\section{INTRODUCTION}

The fully implicit/backward Euler method is frequently used to compute an approximation for the time dependent viscous flow problems [1,2]. This is because the method is A-stable, converges to actual solution very fast, and is implemented easily. However, it may not reflect the true physical behavior of the analytical solution in many situations [3]. In a recent paper [4], a numerical approach for ordinary differential equations which uses the time filter was introduced to increase the accuracy of the $\mathrm{BE}$ method, and the idea was applied to the incompressible Navier-Stokes equations (NSE) in [5]. The key idea in [5] is to combine the standard BE time-stepping scheme for the NSE with a linear time filter. Hence, the resulting algorithm requires two uncoupled steps at each time level. The first step calculates an intermediate BE velocity approximation for the NSE, and the second step post proceeds this intermediate velocity by using a second order, linear time filter. By this way, the accuracy of the method is increased from the first order to the second order. Assuming that the time step is constant, the method becomes more attractive because it needs only one line additional code into BE-solver at each time level. In addition to this, eliminating the intermediate step leads to an equivalent method, which is 2-step, Astable but is still of second-order accuracy, ands implifies the stability/the convergence analysis.

The aim of this report is to apply this novel idea to the velocity-vorticity-temperature (VVT) model of time-dependent incompressible, non-isothermal fluid flows [6,7]. VVT model for the incompressible, non-isothermal fluid flowsis given as follows: for a given force field $f:\left(0, t^{*}\right] \times \Omega \rightarrow \mathbb{R}^{d}$, find velocity, pressure, temperature and vorticity fields, i.e., $\mathbf{u}:\left(0, t^{*}\right] \times \Omega \rightarrow \mathbb{R}^{d}$ and $p, \theta, w:\left(0, t^{*}\right] \times \Omega \rightarrow \mathbb{R}$, such that $(\mathbf{u}, P, \theta, w)$ satisfies the equations in $\left(0, t^{*}\right] \times \Omega$ 


$$
\begin{array}{ll}
\frac{\partial \mathbf{u}}{\partial t}-v \Delta \mathbf{u}+w \times \mathbf{u}+\nabla P & =R i T \widehat{\boldsymbol{k}}+f \\
\nabla \cdot \mathbf{u} & =0 \\
\frac{\partial T}{\partial t}-\kappa \Delta T+(\mathbf{u} \cdot \nabla) T & =\gamma \\
\frac{\partial w}{\partial t}-v \Delta w+(\mathbf{u} \cdot \nabla) w & =R i \frac{\partial T}{\partial x}+\nabla \times f .
\end{array}
$$

where $w:=\nabla \times \mathbf{u}$ represents the vorticity of the fluid, $P:=\frac{1}{2}|\mathbf{u}|^{2}+p$ is the Bernoulli pressure.

Moreover, $\widehat{\boldsymbol{k}}$ is the unit vector in the direction of gravity, $v:=1 / \operatorname{Re}$ is the dimensionless kinematic viscosity, where $R e$ denotes the Reynolds number, $R i:=G r / R e^{2}$ is the Richardson number, and $\kappa:=$ $1 /(\operatorname{Pr} R e)$ is thermal diffusivity coefficient.

Velocity-vorticity (VV) formulations have been studied frequently [8-13]. This is due to the fact that the vorticity has an essential role in determining the fluid dynamics. In addition, $(w \times \mathbf{u}, \mathbf{u})=0$, which ensures the energy inequality of the model, and thus the stability of any numerical method for VV is guaranteed.

Due to the success of VV-model in simulations of the incompressible flows, especially for vortex dominated or strongly rotating flows, this report proposes a numerical method to approximate VVTmodel in $2 D$. We make a note here that analyzing the vortex stretching term in $3 D$-setting is very complex. Therefore, VVT-modelis analyzed in $2 D$.

The proposed numerical method to approximate the solutions of VVTconsists of two decoupled steps as in [5]. The first step calculates velocity/vorticity/temperature approximations of the VVT-scheme with the usual BE-FE discretization. The second step introduces three decoupled time filter steps for each of these approximations. Eliminating the intermediate velocity/temperature/vorticity step together with assuming the time step is constant, the proposed method can be reduced to an equivalent algorithm that alike linearly extrapolated, two-step BDF-method (BDF2LE). This makes simpler the stability and error analysis of the numerical method. The time discretization in the proposed algorithm is done such that the convective terms of evolution equations are linear with respect to the first variable.Hence, the whole algorithm becomes linear at each time level. We make a note here that the application of the second order scheme for multiphysics flow problems can be seen in $[14,15]$.

We organize the paper as follows. All necessary mathematical preliminaries are collected in Section 2. Section 3 presents a numerical algorithm for VVT scheme, and studies its stability and convergence to VVT model. We prove that approximate solutions are unconditionally stable according to time step, and converges to analytical solutions of VVT optimally. Section 4 provides two numerical experimentsfor the method. The first one verifies the theoretical convergence rates, and shows that the error between the vorticity and velocity goes to zero. The second numerical experiment tests the method on a benchmark problem, named Marsigli's flow problem. The conclusions of the study are summarized in Section 5.

\section{MATHEMATICAL PRELIMINARIES}

This section is devoted to presenting mathematical preliminaries and notation used throughout this paper. The norms in $H^{k}(\Omega)$ will be denoted by $\|\cdot\|_{k}$, and the norms in Lebesgue spaces by $L^{p}(\Omega), 1 \leq$ $p<\infty$ by $\|\cdot\|_{L} p$, and by $\|\cdot\|_{\infty}$ for $p=\infty$, [16]. Vector valued functions spaces will be indicated with bold letters.

The velocity, pressure and temperature spaces in the periodic setting for Boussinesq system are given by 


$$
\begin{gathered}
\boldsymbol{X}:=\boldsymbol{H}_{\#}^{1}(\Omega)=\left\{v \in \boldsymbol{H}_{l o c}^{1}(\mathbb{R}): \text { v is } 2 \pi \text { periodic in each direction, } \int_{D} \boldsymbol{v} \boldsymbol{d} \boldsymbol{x}=\mathbf{0}\right\}, \\
\mathrm{Q}:=L_{\#}^{2}(\Omega)=\left\{q \in L_{\text {loc }}^{2}(\mathbb{R}): \text { is } 2 \pi \text { periodic in each direction, } \int_{D} \mathrm{q} \mathrm{dx}=0\right\}, \\
Y:=H_{\#}^{1}(\Omega)=\left\{v \in H_{l o c}^{1}(\mathbb{R}): \text { v is } 2 \pi \text { periodic in each direction, } \int_{D} \mathrm{v} \mathrm{dx}=0\right\},
\end{gathered}
$$

where $\Omega$ is a polygonal or polyhedral domain in $\mathbb{R}^{d}(d=2,3)$. We make a note here that we will denote vorticity space as $Y=H_{\#}^{1}(\Omega)$ since vorticity is a scalar quantity in two dimensions. The divergence freesubspace of $\mathbf{X}$ is represented by $\mathbf{V}$, i.e.,

$$
\mathbf{V}:=\{\mathbf{v} \in \mathbf{X}: \nabla \cdot \mathbf{v}=0\} .
$$

The skew symmetrized trilinear form is defined by

$$
b^{*}(\mathbf{u}, \varphi, s):=\frac{1}{2}((\mathbf{u} \cdot \nabla \varphi, s)-(\mathbf{u} \cdot \nabla s, \varphi)), \quad \forall \mathbf{u} \in \mathbf{X}, \forall \varphi, s \in Y .
$$

Notice that $b^{*}(\mathbf{u}, \varphi, s)=0$. This operator satisfies the following [17].

Lemma 1. Let $\boldsymbol{u} \in \boldsymbol{X}$ and $\varphi, s \in Y$. Then there exists a constant $C:=C(\Omega)$ such that it holds

$$
\begin{aligned}
& b^{*}(\mathbf{u}, \theta, S) \leq C\|\nabla \mathbf{u}\|\|\nabla \theta\|\|\nabla S\|, \\
& b^{*}(\mathbf{u}, \theta, S) \leq C\|\mathbf{u}\|^{1 / 2}\|\nabla \mathbf{u}\|^{1 / 2}\|\nabla \theta\|\|\nabla S\|, \\
& b^{*}(\mathbf{u}, \theta, S) \leq C\|\mathbf{u}\|\|\theta\|_{2}\|\nabla S\| .
\end{aligned}
$$

$\mathbb{S}_{h}$ stands for a decomposition of a domain $\Omega$ into triangles in $2 d$ or tetrahedron in $3 d$ with maximum diameter $h$, and associated velocity, pressure and temperature/vorticity finite element (FE) spaces are denoted by $\mathbf{X}_{h} \subset \mathbf{X}, Q_{h} \subset Q$, and $Y_{h} \subset Y$ which satisfy approximation properties of piecewise polynomials of local degree $k+1, k-1$ and $k$, respectively :

$$
\begin{gathered}
\inf _{\mathbf{v}_{h} \in \mathbf{X}_{h}}\left\{\left\|\mathbf{u}-\mathbf{v}_{h}\right\|+h\left\|\nabla\left(\mathbf{u}-\mathbf{v}_{h}\right)\right\|\right\} \leq C h^{k+2}\|\mathbf{u}\|_{k+2}, \\
\inf _{r_{h} \in Q_{h}}\left\|p-r_{h}\right\| \leq C h^{k}\|p\|_{k}, \\
\inf _{w_{h} \in Y_{h}}\left\{\left\|w-w_{h}\right\|+h\left\|\nabla\left(w-w_{h}\right)\right\|\right\} \leq C h^{k+1}\|w\|_{k+1},
\end{gathered}
$$

for all $\mathbf{u} \in \mathbf{X} \cap \mathbf{H}^{k+1}(\Omega), p \in Q \cap H^{k}(\Omega)$ and $w \in Y \cap H^{k+1}(\Omega)$. Furthermore, assume $\left(\mathbf{X}_{h}, Q_{h}\right)$ satisfies the discrete inf-sup stability (LBB) condition, see [14]

$$
\inf _{q_{h} \in Q_{h \mathbf{v}_{h} \in \mathbf{X}_{h}}} \frac{\left(q_{h}, \nabla \cdot \mathbf{v}_{h}\right)}{\left\|q_{h}\right\|\left\|\nabla \mathbf{v}_{h}\right\|} \geq \beta>0,
$$

where $\beta$ is a constant independent of $h$. It is well known that the LBB condition assures that the approximation properties of the spaces $\mathbf{X}_{h}$ and $\mathbf{V}_{h}$ are equivalent in those of space $\mathbf{V}$ :

$$
\inf _{\mathbf{v}_{h} \in \mathbf{V}_{h}}\left\|\nabla\left(\mathbf{u}-\mathbf{v}_{h}\right)\right\| \leq C_{\mathbf{v}_{h} \in \mathbf{X}_{h}}\left\|\nabla\left(\mathbf{u}-\mathbf{v}_{h}\right)\right\|, \quad \forall \mathbf{u} \in \mathbf{V},
$$

where $\mathbf{V}_{h}$ is discretely divergence-free subspace

$$
\mathbf{V}_{h}:=\left\{\mathbf{v}_{h} \in \mathbf{X}_{h}:\left(q_{h}, \nabla \cdot \mathbf{v}_{h}\right)=0, \quad \forall q_{h} \in Q_{h}\right\} .
$$


Let $\varphi(t, \mathbf{x})$ be defined on $\left(0, t^{*}\right]$. Then we introduce the following norms

$$
\|\varphi\|_{\infty, k}:=e s \sup _{0<t<t^{*}}\|\varphi(t, \cdot)\|_{k} \text {, and }\|\varphi\|_{m, k}:=\left(\int_{0}^{t^{*}}\|\varphi(t, \cdot)\|_{k}^{m} \mathrm{dt}\right)^{1 / m}, 1 \leq m<\infty .
$$

Introducing the notationt $t^{n+1}:=(n+1) \Delta t$, where $\Delta t$ is a chosen time-step, we indicate $\varphi^{n+1}:=$ $\varphi\left(t^{n+1}\right)$, and use the following discrete time norms:

$$
\||\varphi|\|_{\infty, k}:=\max _{0 \leq n \leq N}\left\|\varphi\left(t^{n}, \cdot\right)\right\|_{k} \text { and }\||\varphi|\|_{m, k}:=\left(\Delta t \sum_{n=0}^{N-1}\|\varphi(t, \cdot)\|_{k}^{m}\right)^{\frac{1}{m}}
$$

The difference and interpolation operators are defined as

Notice that

$$
\delta\left[\Phi^{n+1}\right]:=\frac{3}{2} \Phi^{n+1}-2 \Phi^{n}+\frac{1}{2} \theta^{n-1}, \quad E\left[\Phi^{n+1}\right]:=\frac{3}{2} \Phi^{n+1}-\Phi^{n}+\frac{1}{2} \Phi^{n-1} .
$$

$$
\frac{\delta\left[\Phi^{n+1}\right]}{\Delta t}=\Phi_{t}^{n+1}+\mathcal{O}\left(\Delta t^{2}\right) \text {, and } E\left[\Phi^{n+1}\right]=\Phi^{n+1}+\mathcal{O}\left(\Delta t^{2}\right)
$$

Using Taylor expansion with integral reminder term, one can have the following.

Lemma 2. Assume $\Phi$ sufficiently smooth. Then, the following holds [5]

$$
\begin{gathered}
\left\|\Phi_{t}^{n+1}-\frac{\delta\left[\Phi^{n+1}\right]}{\Delta t}\right\|^{2} \leq \frac{6}{5} \Delta t^{3} \int_{t^{n-1}}^{t^{n+1}}\left\|\Phi_{t t t}\right\|^{2} d t, \\
\left\|\Phi^{n+1}-E\left[\Phi^{n+1}\right]\right\|^{2} \leq \frac{\Delta t^{3}}{6} \int_{t^{n-1}}^{t^{n+1}}\left\|\Phi_{t t}\right\|^{2} d t, \\
\left\|\Phi^{n+1}-2 \Phi^{n}+\Phi^{n-1}\right\|^{2} \leq \frac{\Delta t^{3}}{3} \int_{t^{n-1}}^{t^{n+1}}\left\|\Phi_{t t}\right\|^{2} d t .
\end{gathered}
$$

In the stability and convergence analysis, we often call the Poincaré-Friedrichs' and Young's inequalities.

Lemma 3. (The Poincaré-Friedrichs' Inequality) For any $\varphi \in X$, there exists a constant $C_{P}$ dependent only on the domain $\Omega$ such that

$$
\|\boldsymbol{\varphi}\|_{L^{2}} \leq C_{P}\|\nabla \boldsymbol{\varphi}\|_{L^{2}} .
$$

Lemma 4. (The Young's Inequality) Let $\lambda, \alpha$ be non-negative real numbers. Then for any $\varepsilon>0$

where $\frac{1}{p}+\frac{1}{q}=1$ with $p, q \in[1, \infty)$.

$$
\lambda \alpha \leq \frac{\varepsilon}{p} \lambda^{p}+\frac{\varepsilon^{-q / p}}{q} \alpha^{q},
$$

In the last step of the convergence analysis, a different version of the usual discrete Gronwall's Lemma will be used, see e.g.,[12].

Lemma 5. (Discrete Gronwall's Lemma) Let $\Delta t, B$ and $a_{n}, b_{n}, c_{n}, d_{n}$ be finite non negative numbers such that

Then for all $\Delta t>0$,

$$
a_{N}+\Delta t \sum_{n=0}^{N} b_{n} \leq \Delta t \sum_{n=0}^{N-1} d_{n} a_{n}+\Delta t \sum_{n=0}^{N} c_{n}+B \quad \text { for } \quad N \geq 1 .
$$

$$
a_{N}+\Delta t \sum_{n=0}^{N} b_{n} \leq \exp \left(\Delta t \sum_{n=0}^{N-1} d_{n}\right)\left(\Delta t \sum_{n=0}^{N} c_{n}+B\right) \text { for } \quad N \geq 1 .
$$

\section{NUMERICAL ALGORITHMS AND STABILITY}

In this section, we present a time accurate, adaptive discretization for (1). 
Algorithm 1. Let body forces $f, \gamma$, initial velocities and temperatures be given. Select an end time $t^{*}$ and a time step $\Delta t>0$ such that $t^{*}=N \Delta t$. Then for any $n \geq 1$ find $\left(\boldsymbol{u}_{h}^{n+1}, p_{h}^{n+1}, T_{h}^{n+1}, w_{h}^{n+1}\right) \in$ $\left(\boldsymbol{X}_{h}, Q_{h}, Y_{h}, Y_{h}\right)$ via the following two steps:

Step 1:Find $\left(\widehat{\mathbf{u}}_{h}^{n+1}, P_{h}^{n+1}, \widehat{T}_{h}^{n+1}, \widehat{w}_{h}^{n+1}\right) \in\left(\mathbf{X}_{h}, Q_{h}, Y_{h}, Y_{h}\right)$ such that it holds, $\forall\left(\mathbf{v}_{h}, q_{h}, \chi_{h}, s_{h}\right) \in$ $\left(\mathbf{X}_{h}, Q_{h}, Y_{h}, Y_{h}\right)$

$$
\begin{gathered}
\left(\frac{\widehat{\mathbf{u}}_{h}^{n+1}-\mathbf{u}_{h}^{n}}{\Delta t}, \mathbf{v}_{h}\right)+v\left(\nabla \widehat{\mathbf{u}}_{h}^{n+1}, \nabla \mathbf{v}_{h}\right)+\left(\left(2 w_{h}^{n}-w_{h}^{n-1}\right) \times \widehat{\mathbf{u}}_{h}^{n+1}, \mathbf{v}_{h}\right)-\left(P_{h}^{n+1}, \nabla \cdot \mathbf{v}_{h}\right) \\
=\left(\mathbf{f}, \mathbf{v}_{h}\right)+\operatorname{Ri}\left(\left(2 T_{h}^{n}-T_{h}^{n-1}\right) \widehat{\boldsymbol{k}}, \mathbf{v}_{h}\right) \\
\left(\nabla \cdot \widehat{\mathbf{u}}_{h}^{n+1}, q_{h}\right)=0, \\
\left(\frac{\widehat{T}_{h}^{n+1}-T_{h}^{n}}{\Delta t}, \chi_{h}\right)+\kappa\left(\nabla \widehat{T}_{h}^{n+1}, \nabla \chi_{h}\right)+b^{*}\left(2 \mathbf{u}_{h}^{n}-\mathbf{u}_{h}^{n-1}, \widehat{T}_{h}^{n+1}, \chi_{h}\right)=\left(\gamma^{n+1}, \chi_{h}\right), \\
\left(\frac{\widehat{w}_{h}^{n+1}-w_{h}^{n}}{\Delta t}, s_{h}\right)+v\left(\nabla \widehat{w}_{h}^{n+1}, \nabla s_{h}\right)+b^{*}\left(2 \mathbf{u}_{h}^{n}-\mathbf{u}_{h}^{n-1}, \widehat{w}_{h}^{n+1}, s_{h}\right)=\left(\nabla \times \mathbf{f}^{n+1}, s_{h}\right) \\
+R i\left(\frac{\partial\left(2 T_{h}^{n}-T_{h}^{n-1}\right)}{\partial x}, s_{h}\right) .
\end{gathered}
$$

Step 2: Compute $\mathbf{u}_{h}^{n+1} \in \mathbf{X}_{h}, T_{h}^{n+1}, w_{h}^{n+1} \in Y_{h}$ satisfying for each $\mathbf{v}_{h} \in \mathbf{X}_{h}, \chi_{h}, s_{h} \in Y_{h}$

$$
\begin{aligned}
& \left(\mathbf{u}_{h}^{n+1}, \mathbf{v}_{h}\right)=\left(\widehat{\mathbf{u}}_{h}^{n+1}, \mathbf{v}_{h}\right)-\frac{1}{3}\left(\widehat{\mathbf{u}}_{h}^{n+1}-2 \mathbf{u}_{h}^{n}+\mathbf{u}_{h}^{n-1}, \mathbf{v}_{h}\right), \\
& \left(T_{h}^{n+1}, \chi_{h}\right)=\left(\widehat{T}_{h}^{n+1}, \chi_{h}\right)-\frac{1}{3}\left(\widehat{T}_{h}^{n+1}-2 T_{h}^{n}+T_{h}^{n-1}, \chi_{h}\right), \\
& \left(w_{h}^{n+1}, s_{h}\right)=\left(\widehat{w}_{h}^{n+1}, s_{h}\right)-\frac{1}{3}\left(\widehat{w}_{h}^{n+1}-2 w_{h}^{n}+w_{h}^{n-1}, s_{h}\right) .
\end{aligned}
$$

Assuming the time step is constant, using the notations of difference and interpolation operators in place, and inserting

$$
\begin{aligned}
\left(\widehat{\mathbf{u}}_{h}^{n+1}, \mathbf{v}_{h}\right) & =\frac{1}{2}\left(3 \mathbf{u}_{h}^{n+1}-2 \mathbf{u}_{h}^{n}+\mathbf{u}_{h}^{n-1}, \mathbf{v}_{h}\right), \\
\left(\widehat{T}_{h}^{n+1}, \chi_{h}\right) & =\frac{1}{2}\left(3 T_{h}^{n+1}-2 T_{h}^{n}+T_{h}^{n-1}, \chi_{h}\right), \\
\left(\widehat{w}_{h}^{n+1}, s_{h}\right) & =\frac{1}{2}\left(3 w_{h}^{n+1}-2 w_{h}^{n}+w_{h}^{n-1}, s_{h}\right),
\end{aligned}
$$

in the first step of Algorithm 3.1, the following equivalence method is obtained

$$
\begin{array}{r}
\left(\frac{\delta\left[\mathbf{u}_{h}^{n+1}\right]}{\Delta t}, \mathbf{v}_{h}\right)+v\left(\nabla E\left[\mathbf{u}_{h}^{n+1}\right], \nabla \mathbf{v}_{h}\right)+\left(\left(2 w_{h}^{n}-w_{h}^{n-1}\right) \times E\left[\mathbf{u}_{h}^{n+1}\right], \mathbf{v}_{h}\right)-\left(P_{h}^{n+1}, \nabla \cdot \mathbf{v}_{h}\right) \\
=R i\left(\left(2 T_{h}^{n}-T_{h}^{n-1}\right) \widehat{\boldsymbol{k}}, \mathbf{v}_{h}\right)+\left(\mathbf{f}^{n+1}, \mathbf{v}_{h}\right), \\
\left(\nabla \cdot E\left[\mathbf{u}_{h}^{n+1}\right], q_{h}\right)=0, \\
\left(\frac{\delta\left[T_{h}^{n+1}\right]}{\Delta t}, \chi_{h}\right)+\kappa\left(\nabla E\left[T_{h}^{n+1}\right], \nabla \chi_{h}\right)+b^{*}\left(2 \mathbf{u}_{h}^{n}-\mathbf{u}_{h}^{n-1}, E\left[T_{h}^{n+1}\right], \chi_{h}\right)=\left(\gamma^{n+1}, \chi_{h}\right), \\
\left(\frac{\delta\left[w_{h}^{n+1}\right]}{\Delta t}, s_{h}\right)+v\left(\nabla E\left[w_{h}^{n+1}\right], \nabla s_{h}\right)+b^{*}\left(2 \mathbf{u}_{h}^{n}-\mathbf{u}_{h}^{n-1}, E\left[w_{h}^{n+1}\right], s_{h}\right)= \\
\left(\nabla \times \mathbf{f}^{n+1}, \mathbf{v}_{h}\right) \\
+R i\left(\frac{\partial\left(2 T_{h}^{n}-T_{h}^{n-1}\right)}{\partial x}, s_{h}\right) .
\end{array}
$$

Remark 6. Notice that time derivative terms are dicretizatized by using BDF2-time stepping method, but the remaining terms are not. Therefore, the method is not the standard BDF2LE-method.

We now prove that the approximate solutions to Algorithm 1 are stable without any time step restriction.

Lemma 7. Assume $f \in L^{\infty}\left(0, t^{*} ; \boldsymbol{L}^{2}(\Omega)\right)$, and $\gamma \in L^{\infty}\left(0, t^{*} ; H^{-1}(\Omega)\right)$. Then for any $\Delta t>0$, solutions to Algorithm 1 satisfy the bound 


$$
\begin{gathered}
\left\|T_{h}^{N}\right\|^{2}+\left\|2 T_{h}^{N}-T_{h}^{N-1}\right\|^{2}+\left\|T_{h}^{N}-T_{h}^{N-1}\right\|^{2}+3 \sum_{n=0}^{N-1}\left\|T_{h}^{n+1}-2 T_{h}^{n}+T_{h}^{n-1}\right\|^{2} \\
+2 \kappa \Delta t \sum_{n=0}^{N-1}\left\|\nabla E\left[T_{h}^{n+1}\right]\right\|^{2} \leq\left\|T_{h}^{1}\right\|^{2}+\left\|2 T_{h}^{1}-T_{h}^{0}\right\|^{2}+\left\|T_{h}^{1}-T_{h}^{0}\right\|^{2} \\
+2 \kappa^{-1} \Delta t \sum_{n=0}^{N-1}\left\|\gamma^{n+1}\right\|^{2}=: C_{T}^{2}
\end{gathered}
$$

and

$$
\begin{array}{r}
\left\|\mathbf{u}_{h}^{N}\right\|^{2}+\left\|2 \mathbf{u}_{h}^{N}-\mathbf{u}_{h}^{N-1}\right\|^{2}+\left\|\mathbf{u}_{h}^{N}-\mathbf{u}_{h}^{N-1}\right\|^{2}+3 \sum_{n=0}^{N-1}\left\|\mathbf{u}_{h}^{n+1}-2 \mathbf{u}_{h}^{n}+\mathbf{u}_{h}^{n-1}\right\|^{2} \\
+2 v \Delta t \sum_{n=0}^{N-1}\left\|\nabla E\left[\mathbf{u}_{h}^{n+1}\right]\right\|^{2} \leq\left\|\mathbf{u}_{h}^{1}\right\|^{2}+\left\|2 \mathbf{u}_{h}^{1}-\mathbf{u}_{h}^{0}\right\|^{2}+\left\|\mathbf{u}_{h}^{1}-\mathbf{u}_{h}^{0}\right\|^{2} \\
+4 v^{-1} R i^{2} C_{P}^{2} C_{T}^{2} t^{*}+4 v^{-1} \Delta t \sum_{n=0}^{N-1}\left\|f^{n+1}\right\|_{-1}^{2},
\end{array}
$$

and

$$
\begin{array}{r}
\left\|w_{h}^{N}\right\|^{2}+\left\|2 w_{h}^{N}-w_{h}^{N-1}\right\|^{2}+\left\|w_{h}^{N}-T_{h}^{N-1}\right\|^{2}+3 \sum_{n=0}^{N-1}\left\|w_{h}^{n+1}-2 w_{h}^{n}+w_{h}^{n-1}\right\|^{2} \\
+2 v \Delta t \sum_{n=0}^{N-1}\left\|\nabla E\left[w_{h}^{n+1}\right]\right\|^{2} \leq\left\|w_{h}^{1}\right\|^{2}+\left\|2 w_{h}^{1}-w_{h}^{0}\right\|^{2}+\left\|w_{h}^{1}-w_{h}^{0}\right\|^{2} \\
+4 v^{-1} R i^{2} C_{T}^{2} t^{*}+4 v^{-1} \Delta t \sum_{n=0}^{N-1}\left\|f^{n+1}\right\|^{2} .
\end{array}
$$

Proof. Setting $\mathbf{v}_{h}=E\left[\mathbf{u}_{h}^{n+1}\right], q_{h}=P_{h}^{n+1}, \chi_{h}=E\left[T_{h}^{n+1}\right]$ and $s_{h}=E\left[w_{h}^{n+1}\right]$ in (6)-(9) vanishes nonlinear and the pressure terms. Then using the algebraic identity

$$
\begin{gathered}
\left(\frac{3 a-4 b+c}{2}\right)\left(\frac{3 a-2 b+c}{2}\right) \\
=\frac{1}{4}\left[a^{2}+(2 a-b)^{2}+(a-b)^{2}\right]-\frac{1}{4}\left[b^{2}+(2 b-c)^{2}+(b-c)^{2}\right]+\frac{3}{4}(a-2 b+c)^{2}
\end{gathered}
$$

yields

$$
\begin{aligned}
& \frac{1}{4 \Delta t}\left[\left\|\mathbf{u}_{h}^{n+1}\right\|^{2}+\left\|2 \mathbf{u}_{h}^{n+1}-\mathbf{u}_{h}^{n}\right\|^{2}+\left\|\mathbf{u}_{h}^{n+1}-\mathbf{u}_{h}^{n}\right\|^{2}\right] \\
& -\frac{1}{4 \Delta t}\left[\left\|\mathbf{u}_{h}^{n}\right\|^{2}+\left\|2 \mathbf{u}_{h}^{n}-\mathbf{u}_{h}^{n-1}\right\|^{2}+\left\|\mathbf{u}_{h}^{n}-\mathbf{u}_{h}^{n-1}\right\|^{2}\right]+\frac{3}{4 \Delta t}\left\|\mathbf{u}_{h}^{n+1}-2 \mathbf{u}_{h}^{n}+\mathbf{u}_{h}^{n-1}\right\|^{2} \\
& +v\left\|\nabla E\left[\mathbf{u}_{h}^{n+1}\right]\right\|^{2} \leq R i\left|\left(\left(2 T_{h}^{n}-T_{h}^{n-1}\right) \widehat{\boldsymbol{k}}, E\left[\mathbf{u}_{h}^{n+1}\right]\right)\right|+\left|\left(f^{n+1}, E\left[\mathbf{u}_{h}^{n+1}\right]\right)\right|,
\end{aligned}
$$

and

$$
\begin{aligned}
& \frac{1}{4 \Delta t}\left[\left\|T_{h}^{n+1}\right\|^{2}+\left\|2 T_{h}^{n+1}-T_{h}^{n}\right\|^{2}+\left\|T_{h}^{n+1}-T_{h}^{n}\right\|^{2}\right] \\
& -\frac{1}{4 \Delta t}\left[\left\|T_{h}^{n}\right\|^{2}+\left\|2 T_{h}^{n}-T_{h}^{n-1}\right\|^{2}+\left\|T_{h}^{n}-T_{h}^{n-1}\right\|^{2}\right]+\frac{3}{4 \Delta t}\left\|T_{h}^{n+1}-2 T_{h}^{n}+T_{h}^{n-1}\right\|^{2} \\
& +\kappa\left\|\nabla E\left[T_{h}^{n+1}\right]\right\|^{2} \leq\left|\left(\gamma^{n+1}, E\left[T_{h}^{n+1}\right]\right)\right|,
\end{aligned}
$$

and

$$
\begin{aligned}
& \frac{1}{4 \Delta t}\left[\left\|w_{h}^{n+1}\right\|^{2}+\left\|2 w_{h}^{n+1}-w_{h}^{n}\right\|^{2}+\left\|w_{h}^{n+1}-w_{h}^{n}\right\|^{2}\right] \\
& -\frac{1}{4 \Delta t}\left[\left\|w_{h}^{n}\right\|^{2}+\left\|2 w_{h}^{n}-w_{h}^{n-1}\right\|^{2}+\left\|w_{h}^{n}-w_{h}^{n-1}\right\|^{2}\right]+\frac{3}{4 \Delta t}\left\|w_{h}^{n+1}-2 w_{h}^{n}+w_{h}^{n-1}\right\|^{2}
\end{aligned}
$$


$+v\left\|\nabla E\left[w_{h}^{n+1}\right]\right\|^{2} \leq R i\left|\left(\frac{\partial\left(2 T_{h}^{n}-T_{h}^{n-1}\right)}{\partial x}, E\left[w_{h}^{n+1}\right]\right)\right|+\left|\left(\nabla \times \mathbf{f}^{n+1}, E\left[w_{h}^{n+1}\right]\right)\right|$.

Applying the Cauchy-Schwarz, Lemma 3, Lemma 4 to the right hand isde of (11)-(12) yields

$$
\begin{aligned}
R i\left|\left(\left(2 T_{h}^{n}-T_{h}^{n-1}\right) \widehat{\boldsymbol{k}}, E\left[\mathbf{u}_{h}^{n+1}\right]\right)\right| \leq R i\left\|2 T_{h}^{n}-T_{h}^{n-1}\right\| C_{P}\left\|\nabla E\left[\mathbf{u}_{h}^{n+1}\right]\right\| \\
\leq v^{-1} R i^{2} C_{P}^{2}\left\|2 T_{h}^{n}-T_{h}^{n-1}\right\|^{2}+\frac{v}{4}\left\|\nabla E\left[\mathbf{u}_{h}^{n+1}\right]\right\|^{2}, \\
\left|\left(f^{n+1}, E\left[\mathbf{u}_{h}^{n+1}\right]\right)\right| \leq v^{-1}\left\|f^{n+1}\right\|_{-1}^{2}+\frac{v}{4}\left\|\nabla E\left[\mathbf{u}_{h}^{n+1}\right]\right\|^{2}, \\
\left|\left(\gamma^{n+1}, E\left[T_{h}^{n+1}\right]\right)\right| \leq \frac{\kappa^{-1}}{2}\left\|\gamma^{n+1}\right\|_{-1}^{2}+\frac{\kappa}{2}\left\|\nabla E\left[T_{h}^{n+1}\right]\right\|^{2} .
\end{aligned}
$$

To bound the terms on the right hand side of (13), use integration by parts and the standard inequalities to get

$$
\begin{aligned}
R i\left|\left(\frac{\partial\left(2 T_{h}^{n}-T_{h}^{n-1}\right)}{\partial x}, E\left[w_{h}^{n+1}\right]\right)\right| & \leq R i\left\|2 T_{h}^{n}-T_{h}^{n-1}\right\|\left\|\nabla E\left[w_{h}^{n+1}\right]\right\| \\
& \leq v^{-1} R i^{2}\left\|2 T_{h}^{n}-T_{h}^{n-1}\right\|^{2}+\frac{v}{4}\left\|\nabla E\left[w_{h}^{n+1}\right]\right\|^{2}, \\
\left|\left(\nabla \times f^{n+1}, E\left[w_{h}^{n+1}\right]\right)\right| & \leq v^{-1}\left\|f^{n+1}\right\|^{2}+\frac{v}{4}\left\|\nabla E\left[w_{h}^{n+1}\right]\right\|^{2} .
\end{aligned}
$$

Inserting all these estimates, multiplying by $4 \Delta t$ and summing over time steps produces

$$
\begin{array}{r}
\left\|\mathbf{u}_{h}^{N}\right\|^{2}+\left\|2 \mathbf{u}_{h}^{N}-\mathbf{u}_{h}^{N-1}\right\|^{2}+\left\|\mathbf{u}_{h}^{N}-\mathbf{u}_{h}^{N-1}\right\|^{2}+3 \sum_{n=0}^{N-1}\left\|\mathbf{u}_{h}^{n+1}-2 \mathbf{u}_{h}^{n}+\mathbf{u}_{h}^{n-1}\right\|^{2} \\
+2 v \Delta t \sum_{n=0}^{N-1}\left\|\nabla E\left[\mathbf{u}_{h}^{n+1}\right]\right\|^{2} \\
\leq\left\|\mathbf{u}_{h}^{1}\right\|^{2}+\left\|2 \mathbf{u}_{h}^{1}-\mathbf{u}_{h}^{0}\right\|^{2}+\left\|\mathbf{u}_{h}^{1}-\mathbf{u}_{h}^{0}\right\|^{2}+4 v^{-1} R i^{2} C_{P}^{2} \Delta t \sum_{n=0}^{N-1}\left\|2 T_{h}^{n}-T_{h}^{n-1}\right\|^{2} \\
+4 v^{-1} \Delta t \sum_{n=0}^{N-1}\left\|f^{n+1}\right\|_{-1}^{2},
\end{array}
$$

and

$$
\begin{gathered}
\left\|T_{h}^{N}\right\|^{2}+\left\|2 T_{h}^{N}-T_{h}^{N-1}\right\|^{2}+\left\|T_{h}^{N}-T_{h}^{N-1}\right\|^{2}+3 \sum_{n=0}^{N-1}\left\|T_{h}^{n+1}-2 T_{h}^{n}+T_{h}^{n-1}\right\|^{2} \\
+2 \kappa \Delta t \sum_{n=0}^{N-1}\left\|\nabla E\left[T_{h}^{n+1}\right]\right\|^{2} \\
\leq\left\|T_{h}^{1}\right\|^{2}+\left\|2 T_{h}^{1}-T_{h}^{0}\right\|^{2}+\left\|T_{h}^{1}-T_{h}^{0}\right\|^{2}+2 \kappa^{-1} \Delta t \sum_{n=0}^{N-1}\left\|\gamma^{n+1}\right\|_{-1}^{2}=: C_{T}^{2}
\end{gathered}
$$

and

$$
\begin{gathered}
\left\|w_{h}^{N}\right\|^{2}+\left\|2 w_{h}^{N}-w_{h}^{N-1}\right\|^{2}+\left\|w_{h}^{N}-w_{h}^{N-1}\right\|^{2}+3 \sum_{n=0}^{N-1}\left\|w_{h}^{n+1}-2 w_{h}^{n}+w_{h}^{n-1}\right\|^{2} \\
+2 v \Delta t \sum_{n=0}^{N-1}\left\|\nabla E\left[w_{h}^{n+1}\right]\right\|^{2}
\end{gathered}
$$

$\leq\left\|w_{h}^{1}\right\|^{2}+\left\|2 w_{h}^{1}-w_{h}^{0}\right\|^{2}+\left\|w_{h}^{1}-w_{h}^{0}\right\|^{2}+4 v^{-1} R i^{2} C_{P}^{2} \Delta t \sum_{n=0}^{N-1}\left\|2 T_{h}^{n}-T_{h}^{n-1}\right\|^{2}$ $+2 v^{-1} \Delta t \sum_{n=0}^{N-1}\left\|f^{n+1}\right\|^{2}$.

The left hand side of (15) is bounded by data. Therefore, the approximate temperature solution is stable. Using temperature stability bound in (14) and (16) yields the desired velocity and vorticity bounds. 


\section{ERROR ANALYSIS}

In this section, we study the convergence of Algorithm 1, and give an error estimate for that. At any time step $t^{n+1}$, true solutions of VVT-model satisfy the following :

$$
\begin{gathered}
\left(\frac{\delta\left[\mathbf{u}^{n+1}\right]}{\Delta t}, \mathbf{v}_{h}\right)+v\left(\nabla E\left[\mathbf{u}^{n+1}\right], \nabla \mathbf{v}_{h}\right)+\left(\left(2 w^{n}-w^{n-1}\right) \times E\left[\mathbf{u}^{n+1}\right], \mathbf{v}_{h}\right)-\left(P^{n+1}, \nabla \cdot \mathbf{v}_{h}\right) \\
=R i\left(\left(2 T^{n}-T^{n-1}\right) \widehat{\boldsymbol{k}}, \mathbf{v}_{h}\right)+\left(f^{n+1}, \mathbf{v}_{h}\right)-\Lambda_{1}\left(\mathbf{u}, T, w, \mathbf{v}_{h}\right) \\
\left(\frac{\delta\left[T^{n+1}\right]}{\Delta t}, \chi_{h}\right)+\kappa\left(\nabla E\left[T^{n+1}\right], \nabla \chi_{h}\right)+b^{*}\left(2 \mathbf{u}^{n}-\mathbf{u}^{n-1}, E\left[T^{n+1}\right], \chi_{h}\right)=\left(\gamma^{n+1}, \chi_{h}\right)-\Lambda_{2}\left(\mathbf{u}, T, \chi_{h}\right)( \\
\left(\frac{\delta\left[w^{n+1}\right]}{\Delta t}, s_{h}\right)+v\left(\nabla E\left[w^{n+1}\right], \nabla s_{h}\right)+b^{*}\left(2 \mathbf{u}^{n}-\mathbf{u}^{n-1}, E\left[w^{n+1}\right], s_{h}\right) \\
=\left(\nabla \times f^{n+1}, s_{h}\right)+R i\left(\frac{\partial\left(2 T^{n}-T^{n-1}\right)}{\partial x}, s_{h}\right)-\Lambda_{3}\left(\mathbf{u}, T, w, \chi_{h}\right)
\end{gathered}
$$

where $\Lambda_{1}\left(\mathbf{u}, T, w, \mathbf{v}_{h}\right), \Lambda_{2}\left(\mathbf{u}, T, \chi_{h}\right)$ and $\Lambda_{3}\left(\mathbf{u}, T, w, s_{h}\right)$ are consistency errors and given by

$$
\begin{aligned}
\Lambda_{1}\left(\mathbf{u}, T, w, \mathbf{v}_{h}\right):=\left(\mathbf{u}_{t}^{n+1}-\right. & \left.\frac{\delta\left[\mathbf{u}^{n+1}\right]}{\Delta t}, \mathbf{v}_{h}\right)+v\left(\nabla\left(\mathbf{u}^{n+1}-E\left[\mathbf{u}^{n+1}\right]\right), \nabla \mathbf{v}_{h}\right)+\left(w^{n+1} \times \mathbf{u}^{n+1}, \mathbf{v}_{h}\right) \\
& -\left(\left(2 w^{n}-w^{n-1}\right) \times E\left[\mathbf{u}^{n+1}\right], \mathbf{v}_{h}\right)-R i\left(\left(T^{n+1}-2 T^{n}+T^{n-1}\right) \widehat{\boldsymbol{k}}, \mathbf{v}_{h}\right),
\end{aligned}
$$

and

$$
\begin{aligned}
\Lambda_{2}\left(\mathbf{u}, T, \chi_{h}\right):=\left(T_{t}^{n+1}-\frac{\delta\left[T^{n+1}\right]}{\Delta t}, \chi_{h}\right)+\kappa\left(\nabla\left(T^{n+1}-E\left[T^{n+1}\right]\right), \nabla \chi_{h}\right) & +b^{*}\left(\mathbf{u}^{n+1}, T^{n+1}, \chi_{h}\right) \\
& -b^{*}\left(2 \mathbf{u}^{n}-\mathbf{u}^{n-1}, E\left[T^{n+1}\right], \chi_{h}\right),
\end{aligned}
$$

and

$$
\begin{aligned}
\Lambda_{3}\left(\mathbf{u}, T, w, s_{h}\right): & =\left(w_{t}^{n+1}-\frac{\delta\left[w^{n+1}\right]}{\Delta t}, s_{h}\right)+v\left(\nabla\left(w^{n+1}-E\left[w^{n+1}\right]\right), \nabla s_{h}\right)+b^{*}\left(\mathbf{u}^{n+1}, w^{n+1}, s_{h}\right) \\
& -b^{*}\left(2 \mathbf{u}^{n}-\mathbf{u}^{n-1}, E\left[w^{n+1}\right], s_{h}\right)-R i\left(\frac{\partial\left(T^{n+1}-2 T^{n}+T^{n-1}\right)}{\partial x}, s_{h}\right) .
\end{aligned}
$$

We assume that true solutions to VVT-model satisfy the following regularity conditions

$\mathbf{u} \in \mathbf{L}^{\infty}\left(0, t^{*} ; \mathbf{H}_{\#}^{3}(\Omega) \cap \mathbf{H}_{\#}^{\mathrm{k}+2}(\Omega)\right), \mathbf{u}_{t t} \in \mathbf{L}^{2}\left(0, t^{*} ; \mathbf{H}_{\#}^{1}(\Omega)\right), \mathbf{u}_{t t t} \in \mathbf{L}^{2}\left(0, t^{*} ; \mathbf{L}_{\#}^{2}(\Omega)\right)$,

$T \in L^{\infty}\left(0, t^{*} ; H_{\#}^{3}(\Omega) \cap H_{\#}^{k+1}(\Omega)\right)$,

$\mathbf{u}_{t t} \in \mathbf{L}^{2}\left(0, t^{*} ; \mathbf{H}_{\#}^{1}(\Omega)\right), \mathbf{u}_{t t t} \in \mathbf{L}^{2}\left(0, t^{*} ; \mathbf{L}_{\#}^{2}(\Omega)\right)$,

$(\nabla \times \mathbf{u}), T \in L^{\infty}\left(0, t^{*} ; H_{\#}^{1}(\Omega)\right),(\nabla \times \mathbf{u})_{t t}, T_{t t} \in L^{2}\left(0, t^{*} ; H_{\#}^{1}(\Omega)\right)$

$(\nabla \times \mathbf{u})_{t t t}, T_{t t t} \in L^{\infty}\left(0, t^{*} ; L_{\#}^{2}(\Omega)\right)$,

and now give consistency error estimates, which are necessary for the convergence theorem.

Lemma 8. [Estimations of the consistency errors] Assume that true solutions $\boldsymbol{u}, P, T, w$ satisfy (20). Then, it holds

$$
\Delta t \sum_{n=0}^{N-1} \Lambda_{1}\left(\mathbf{u}, T, w, \mathbf{v}_{h}\right)
$$


and

$$
\begin{aligned}
\leq & C(\Delta t)^{4}\left(v^{-1}\left\|\mathbf{u}_{t t t}\right\|_{2,0}^{2}+v\left\|\nabla \mathbf{u}_{t t}\right\|_{2,0}^{2}+v^{-1}\left\|T_{t t}\right\|_{2,0}^{2}+v^{-1}\left\|\nabla \mathbf{u}_{t t}\right\|_{2,0}^{2}\||w|\|_{\infty, 0}^{2}\right. \\
& \left.+v^{-1}\left\|w_{t t}\right\|_{2,0}^{2}\||\nabla \mathbf{u}|\|_{\infty, 0}^{2}\right)+\left(\varepsilon_{1}^{*}+\varepsilon_{2}^{*}+\varepsilon_{3}^{*}+\varepsilon_{4}^{*}\right) v \Delta t \sum_{n=0}^{N-1}\left\|\nabla \mathbf{v}_{h}\right\|^{2},
\end{aligned}
$$

and

$$
\Delta t \sum_{n=0}^{N-1} \Lambda_{2}\left(\mathbf{u}, T, \chi_{h}\right)
$$

$$
\begin{aligned}
\leq C(\Delta t)^{4}\left(\kappa^{-1} \|\right. & T_{t t t}\left\|_{2,0}^{2}+\kappa\right\| \nabla T_{t t}\left\|_{2,0}^{2}+\kappa^{-1}\right\| \nabla T_{t t}\left\|_{2,0}^{2}\right\||\nabla \mathbf{u}| \|_{\infty, 0}^{2} \\
& \left.+\kappa^{-1}\left\|\nabla \mathbf{u}_{t t}\right\|_{2,0}^{2}\||\nabla T|\|_{\infty, 0}^{2}\right)+\left(\varepsilon_{5}^{*}+\varepsilon_{6}^{*}+\varepsilon_{7}^{*}\right) \kappa \Delta t \sum_{n=0}^{N-1}\left\|\nabla \chi_{h}\right\|^{2},
\end{aligned}
$$

$$
\begin{gathered}
\Delta t \sum_{n=0}^{N-1} \Lambda_{3}\left(\mathbf{u}, T, w, s_{h}\right) \\
\leq C(\Delta t)^{4}\left(v^{-1}\left\|w_{t t t}\right\|_{2,0}^{2}+v\left\|\nabla w_{t t}\right\|_{2,0}^{2}+v^{-1}\left\|\nabla w_{t t}\right\|_{2,0}^{2}\||\nabla \mathbf{u}|\|_{\infty, 0}^{2}\right. \\
\left.+v^{-1}\left\|\nabla \mathbf{u}_{t t}\right\|_{2,0}^{2}\||\nabla \mathrm{w}|\|_{\infty, 0}^{2}-v^{-1} R i^{2}\left\|T_{t t}\right\|_{2,0}^{2}\right)+\left(\varepsilon_{8}^{*}+\varepsilon_{9}^{*}+\varepsilon_{10}^{*}\right) v \Delta t \sum_{n=0}^{N-1}\left\|\nabla s_{h}\right\|^{2} .
\end{gathered}
$$

Proof. For brevity, we only present the estimation of $\Lambda_{1}\left(\mathbf{u}, T, w, \mathbf{v}_{h}\right)$ since the estimation of $\Lambda_{2}\left(\mathbf{u}, T, \chi_{h}\right)$ and $\Lambda_{3}\left(\mathbf{u}, T, w, \chi_{h}\right)$ proceed in a similar way. For the first two and the last terms of $\Lambda_{1}\left(\mathbf{u}, T, w, \mathbf{v}_{h}\right)$, we apply the Cauchy-Schwarz Inequality, Lemma 3, Lemma 4 together with Lemma 2 to obtain

$$
\begin{aligned}
& \left(\mathbf{u}_{t}^{n+1}-\frac{\delta\left[\mathbf{u}^{n+1}\right]}{\Delta t}, \mathbf{v}_{h}\right) \leq\left\|\mathbf{u}_{t}^{n+1}-\frac{\delta\left[\mathbf{u}^{n+1}\right]}{\Delta t}\right\| C_{P}\left\|\nabla \mathbf{v}_{h}\right\| \\
& \leq \mathrm{C} C_{P}^{2} v^{-1}\left\|\mathbf{u}_{t}^{n+1}-\frac{\delta\left[\mathbf{u}^{n+1}\right]}{\Delta t}\right\|^{2}+\varepsilon_{1}^{*} v\left\|\nabla \mathbf{v}_{h}\right\|^{2} \\
& \leq C v^{-1}(\Delta t)^{3}\left\|\mathbf{u}_{t t t}\right\|_{L^{2}\left(t^{n-1}, t^{n+1} ; L^{2}\right)}^{2}+\varepsilon_{1}^{*} v\left\|\nabla \mathbf{v}_{h}\right\|^{2}, \\
& v\left(\nabla\left(\mathbf{u}^{n+1}-E\left[\mathbf{u}^{n+1}\right]\right), \nabla \mathbf{v}_{h}\right) \leq v\left\|\nabla\left(\mathbf{u}^{n+1}-E\left[\mathbf{u}^{n+1}\right]\right)\right\|\left\|\nabla \mathbf{v}_{h}\right\| \\
& \leq C v(\Delta t)^{3}\left\|\nabla \mathbf{u}_{t t}\right\|_{L^{2}\left(t^{n-1}, t^{n+1} ; L^{2}\right)}^{2}+\varepsilon_{2}^{*} v\left\|\nabla \mathbf{v}_{h}\right\|^{2}, \\
& \operatorname{Ri}\left(\left(T^{n+1}-2 T^{n}+T^{n-1}\right) \widehat{\boldsymbol{k}}, \mathbf{v}_{h}\right) \leq R i\left\|T^{n+1}-2 T^{n}+T^{n-1}\right\| C_{P}\left\|\nabla \mathbf{v}_{h}\right\| \\
& \leq C v^{-1}(\Delta t)^{3}\left\|T_{t t}\right\|_{L^{2}\left(t^{n-1}, t^{n+1} ; L^{2}\right)}^{2}+\varepsilon_{3}^{*} v\left\|\nabla \mathbf{v}_{h}\right\|^{2} \text {. }
\end{aligned}
$$

To bound non-linear terms, first rewrite that as follows

$$
\begin{aligned}
\left(w^{n+1} \times \mathbf{u}^{n+1}, \mathbf{v}_{h}\right)- & \left(\left(2 w^{n}-w^{n-1}\right) \times E\left[\mathbf{u}^{n+1}\right], \mathbf{v}_{h}\right) \\
& =\left(w^{n+1} \times\left(\mathbf{u}^{n+1}-E\left[\mathbf{u}^{n+1}\right]\right), \mathbf{v}_{h}\right)+\left(\left(w^{n+1}-2 w^{n}+w^{n-1}\right) \times E\left[\mathbf{u}^{n+1}\right], \mathbf{v}_{h}\right) .
\end{aligned}
$$

Using the fact that $(\varphi \times \mathbf{u}, \mathbf{v}) \leq C\|\varphi\|\|\nabla \mathbf{u}\|\|\nabla \mathbf{v}\|, \varphi \in Y, \mathbf{u}, \mathbf{v} \in \mathbf{X}$, along with Lemma 1 , and the Young's inequality yields

$$
\begin{aligned}
& \left(w^{n+1} \times\left(\mathbf{u}^{n+1}-E\left[\mathbf{u}^{n+1}\right]\right), \mathbf{v}_{h}\right)+\left(\left(w^{n+1}-2 w^{n}+w^{n-1}\right) \times E\left[\mathbf{u}^{n+1}\right], \mathbf{v}_{h}\right) \\
& \leq C\left(\left\|w^{n+1}\right\|\left\|\nabla\left(\mathbf{u}^{n+1}-E\left[\mathbf{u}^{n+1}\right]\right)\right\|+\left\|w^{n+1}-2 w^{n}+w^{n-1}\right\|\left\|\nabla E\left[\mathbf{u}^{n+1}\right]\right\|\right)\left\|\nabla \mathbf{v}_{h}\right\| \\
& \leq C v^{-1} \Delta t^{3}\left(\left\|w^{n+1}\right\|^{2}\left\|\nabla \mathbf{u}_{t t}\right\|_{L^{2}\left(t^{n-1}, t^{n+1} ; L^{2}\right)}+\left\|w_{t t}\right\|_{L^{2}\left(t^{n-1}, t^{n+1} ; L^{2}\right)}^{2}\left\|\nabla E\left[\mathbf{u}^{n+1}\right]\right\|^{2}\right) \\
& \quad+\varepsilon_{4}^{*} v\left\|\nabla \mathbf{v}_{h}\right\|^{2} .
\end{aligned}
$$

Inserting all these estimates to the right hand side of $\Lambda_{1}\left(\mathbf{u}, T, w, \mathbf{v}_{h}\right)$ produces 


$$
\begin{gathered}
\left|\Lambda_{1}\left(\mathbf{u}, T, w, \mathbf{v}_{h}\right)\right| \\
\leq C(\Delta t)^{3}\left(v^{-1}\left\|\mathbf{u}_{t t t}\right\|_{L^{2}\left(t^{n-1}, t^{n+1} ; L^{2}\right)}^{2}+v\left\|\nabla \mathbf{u}_{t t}\right\|_{L^{2}\left(t^{n-1}, t^{n+1} ; L^{2}\right)}^{2}+v^{-1}\left\|T_{t t}\right\|_{L^{2}\left(t^{n-1}, t^{n+1} ; L^{2}\right)}^{2}\right. \\
\left.+v^{-1}\||w|\|_{\infty, 0}^{2}\left\|\nabla \mathbf{u}_{t t}\right\|_{L^{2}\left(t^{n-1}, t^{n+1} ; L^{2}\right)}^{2}+v^{-1}\left\|w_{t t}\right\|_{L^{2}\left(t^{n-1}, t^{n+1} ; L^{2}\right)}^{2}\|\nabla \mathbf{u} \mid\|_{\infty, 0}^{2}\right) \\
+\left(\varepsilon_{1}^{*}+\varepsilon_{2}^{*}+\varepsilon_{3}^{*}+\varepsilon_{4}^{*}\right) v\left\|\nabla \mathbf{v}_{h}\right\|^{2} .
\end{gathered}
$$

Summing over time steps and multiplying by $\Delta t$ finishes the proof.

We now present the convergence result.

Theorem 9. Assume that true solutions of Algorithm 1 satisfy the regularity conditions (20), and $\boldsymbol{u}_{h}^{n+1}, p_{h}^{n+1}, T_{h}^{n+1}, w_{h}^{n+1}$ be solutions to (6)-(9). Then the errors satisfy the bounds: for any $\Delta t>0$,

$$
\begin{aligned}
& \left\|\mathbf{e}_{\mathbf{u}}^{N}\right\|^{2}+\left\|e_{w}^{N}\right\|^{2}+\left\|e_{T}^{N}\right\|^{2}+\left\|2 \mathbf{e}_{\mathbf{u}}^{N}-\mathbf{e}_{\mathbf{u}}^{N-1}\right\|^{2}+\left\|2 e_{w}^{N}-e_{w}^{N-1}\right\|^{2}+\left\|2 e_{T}^{N}-e_{T}^{N-1}\right\|^{2} \\
& +\left\|\mathbf{e}_{\mathbf{u}}^{N}-\mathbf{e}_{\mathbf{u}}^{N-1}\right\|^{2}+\left\|e_{w}^{N}-e_{w}^{N-1}\right\|^{2}+\left\|e_{T}^{N}-e_{T}^{N-1}\right\|^{2} \\
& +3 \sum_{n=0}^{N-1}\left(\left\|\mathbf{e}_{\mathbf{u}}^{n+1}-2 \mathbf{e}_{\mathbf{u}}^{n}+\mathbf{e}_{\mathbf{u}}^{n-1}\right\|^{2}+\left\|e_{w}^{n+1}-2 e_{w}^{n}+e_{w}^{n-1}\right\|^{2}+\left\|e_{T}^{n+1}-2 e_{T}^{n}+e_{T}^{n-1}\right\|^{2}\right) \\
& +\Delta t \sum_{n=0}^{N-1}\left(v\left\|\nabla E\left[\mathbf{u}^{n+1}\right]\right\|^{2}+v\left\|\nabla E\left[w^{n+1}\right]\right\|^{2}+\kappa\left\|\nabla E\left[T^{n+1}\right]\right\|^{2}\right) \leq C\left(h^{2 k}+\Delta t^{4}\right),
\end{aligned}
$$

where $C$ is a generic constant independent of $h$ and $\Delta t$, and

$$
\mathbf{e}_{\mathbf{u}}^{n+1}:=\mathbf{u}^{n+1}-\mathbf{u}_{h}^{n+1}, e_{T}^{n+1}:=T^{n+1}-T_{h}^{n+1}, e_{w}^{n+1}:=w^{n+1}-w_{h}^{n+1}
$$

are the velocity, temperature and vorticity errors.

Proof. Subtract (6)-(9) from (17)-(19) to get, for any $r_{h} \in Q_{h}$

$$
\begin{aligned}
\left(\frac{\delta\left[\mathbf{e}_{\mathbf{u}}^{n+1}\right]}{\Delta t}, \mathbf{v}_{h}\right)+ & v\left(\nabla E\left[\mathbf{e}_{\mathbf{u}}^{n+1}\right], \nabla \mathbf{v}_{h}\right)-\left(P^{n+1}-r_{h}, \nabla \cdot \mathbf{v}_{h}\right)+\left(\left(2 e_{w}^{n}-e_{w}^{n-1}\right) \times E\left[\mathbf{u}^{n+1}\right], \mathbf{v}_{h}\right) \\
+ & \left(\left(2 w_{h}^{n}-w_{h}^{n-1}\right) \times E\left[\mathbf{e}_{\mathbf{u}}^{n+1}\right], \mathbf{v}_{h}\right)=R i\left(\left(2 e_{T}^{n}-e_{T}^{n-1}\right) \widehat{\boldsymbol{k}}, \mathbf{v}_{h}\right)-\Lambda_{1}\left(\mathbf{u}, T, w, \mathbf{v}_{h}\right), \\
\left(\frac{\delta\left[e_{T}^{n+1}\right]}{\Delta t}, \chi_{h}\right)+ & \kappa\left(\nabla E\left[e_{T}^{n+1}\right], \nabla \chi_{h}\right)+b^{*}\left(2 \mathbf{e}_{\mathbf{u}}^{n}-\mathbf{e}_{\mathbf{u}}^{n-1}, E\left[T^{n+1}\right], \chi_{h}\right) \\
& +b^{*}\left(2 \mathbf{u}_{h}^{n}-\mathbf{u}_{h}^{n-1}, E\left[e_{T}^{n+1}\right], \chi_{h}\right)=-\Lambda_{2}\left(\mathbf{u}, T, \chi_{h}\right), \\
\left(\frac{\delta\left[e_{w}^{n+1}\right]}{\Delta t}, s_{h}\right)+ & v\left(\nabla E\left[e_{w}^{n+1}\right], \nabla s_{h}\right)+b^{*}\left(2 \mathbf{e}_{\mathbf{u}}^{n}-\mathbf{e}_{\mathbf{u}}^{n-1}, E\left[w^{n+1}\right], s_{h}\right) \\
& +b^{*}\left(2 \mathbf{u}_{h}^{n}-\mathbf{u}_{h}^{n-1}, E\left[e_{w}^{n+1}\right], s_{h}\right)=R i\left(\frac{\partial\left(2 e_{T}^{n}-e_{T}^{n-1}\right)}{\partial x}, s_{h}\right)-\Lambda_{3}\left(\mathbf{u}, T, w, s_{h}\right) .
\end{aligned}
$$

Decompose the errors

$$
\begin{aligned}
& \mathbf{e}_{\mathbf{u}}^{n+1}=\left(\mathbf{u}^{n+1}-\widetilde{\mathbf{u}}_{h}^{n+1}\right)-\left(\mathbf{u}_{h}^{n+1}-\widetilde{\mathbf{u}}_{h}^{n+1}\right)=: \eta_{\mathbf{u}}^{n+1}-\boldsymbol{\Psi}_{\mathbf{u}, h}^{n+1}, \\
& e_{T}^{n+1}=\left(T^{n+1}-\widetilde{T}_{h}^{n+1}\right)-\left(T_{h}^{n+1}-\widetilde{T}_{h}^{n+1}\right)=: \eta_{T}^{n+1}-\psi_{T, h}^{n+1}, \\
& e_{w}^{n+1}=\left(w^{n+1}-\widetilde{w}_{h}^{n+1}\right)-\left(w_{h}^{n+1}-\widetilde{w}_{h}^{n+1}\right)=: \eta_{w}^{n+1}-\psi_{w, h}^{n+1},
\end{aligned}
$$

where $\widetilde{\mathbf{u}}_{h}^{n+1}, \widetilde{T}_{h}^{n+1}$ and $\widetilde{w}_{h}^{n+1}$ are the $L^{2}$-orthogonal projections of $\mathbf{u}^{n+1}, T^{n+1}$ and $w^{n+1}$, respectively. Choose $\mathbf{v}_{h}=E\left[\boldsymbol{\psi}_{\mathbf{u}, h}^{n+1}\right]$ in (21), $\chi_{h}=E\left[\psi_{T, h}^{n+1}\right]$ in (22), and $s_{h}=E\left[\psi_{w, h}^{n+1}\right]$ in (23). Using the algebraic identity (10) produces: for any $r_{h} \in Q_{h}$

$$
\begin{aligned}
& \frac{1}{4 \Delta t}\left(\left\|\boldsymbol{\Psi}_{\mathbf{u}, h}^{n+1}\right\|^{2}-\left\|\boldsymbol{\Psi}_{\mathbf{u}, h}^{n}\right\|^{2}\right)+\frac{1}{4 \Delta t}\left(\left\|2 \boldsymbol{\Psi}_{\mathbf{u}, h}^{n+1}-\boldsymbol{\Psi}_{\mathbf{u}, h}^{n}\right\|^{2}-\left\|2 \boldsymbol{\Psi}_{\mathbf{u}, h}^{n}-\boldsymbol{\Psi}_{\mathbf{u}, h}^{n-1}\right\|^{2}\right) \\
& +\frac{1}{4 \Delta t}\left(\left\|\boldsymbol{\Psi}_{\mathbf{u}, h}^{n+1}-\boldsymbol{\Psi}_{\mathbf{u}, h}^{n}\right\|^{2}-\left\|\boldsymbol{\Psi}_{\mathbf{u}, h}^{n}-\boldsymbol{\Psi}_{\mathbf{u}, h}^{n-1}\right\|^{2}\right)+\frac{3}{4 \Delta t}\left\|\boldsymbol{\Psi}_{\mathbf{u}, h}^{n+1}-2 \boldsymbol{\Psi}_{\mathbf{u}, h}^{n}+\boldsymbol{\Psi}_{\mathbf{u}, h}^{n-1}\right\|^{2} \\
& +v\left\|\nabla E\left[\boldsymbol{\Psi}_{\mathbf{u}, h}^{n+1}\right]\right\|^{2} \\
& =v\left(\nabla E\left[\boldsymbol{\eta}_{\mathbf{u}}^{n+1}\right], \nabla E\left[\boldsymbol{\Psi}_{\mathbf{u}, h}^{n+1}\right]\right)-\left(P^{n+1}-r_{h}, \nabla \cdot E\left[\boldsymbol{\Psi}_{\mathbf{u}, h}^{n+1}\right]\right)-\operatorname{Ri}\left(\left(2 e_{T}^{n}-e_{T}^{n-1}\right) \hat{\mathbf{k}}, E\left[\boldsymbol{\Psi}_{\mathbf{u}, h}^{n+1}\right]\right) \\
& +\left(\left(2 e_{w}^{n}-e_{w}^{n-1}\right) \times E\left[\mathbf{u}^{n+1}\right], E\left[\boldsymbol{\Psi}_{\mathbf{u}, h}^{n+1}\right]\right)+\left(\left(2 w_{h}^{n}-w_{h}^{n-1}\right) \times E\left[\boldsymbol{\eta}_{\mathbf{u}}^{n+1}\right], E\left[\boldsymbol{\Psi}_{\mathbf{u}, h}^{n+1}\right]\right)
\end{aligned}
$$


$+\Lambda_{1}\left(\mathbf{u}, T, w, E\left[\Psi_{\mathbf{u}, h}^{n+1}\right]\right)$,

and

$\frac{1}{4 \Delta t}\left(\left\|\psi_{T, h}^{n+1}\right\|^{2}-\left\|\psi_{T, h}^{n}\right\|^{2}\right)+\frac{1}{4 \Delta t}\left(\left\|2 \psi_{T, h}^{n+1}-\psi_{T, h}^{n}\right\|^{2}-\left\|2 \psi_{T, h}^{n}-\psi_{T, h}^{n-1}\right\|^{2}\right)$

$+\frac{1}{4 \Delta t}\left(\left\|\psi_{T, h}^{n+1}-\psi_{T, h}^{n}\right\|^{2}-\left\|\psi_{T, h}^{n}-\psi_{T, h}^{n-1}\right\|^{2}\right)+\frac{3}{4 \Delta t}\left\|\psi_{T, h}^{n+1}-2 \psi_{T, h}^{n}+\psi_{T, h}^{n-1}\right\|^{2}$

$+\kappa\left\|\nabla E\left[\psi_{T, h}^{n+1}\right]\right\|^{2}$

$=\kappa\left(\nabla E\left[\eta_{T}^{n+1}\right], \nabla E\left[\psi_{T, h}^{n+1}\right]\right)+b^{*}\left(2 \mathbf{e}_{\mathbf{u}}^{n}-\mathbf{e}_{\mathbf{u}}^{n-1}, E\left[T^{n+1}\right], E\left[\psi_{T, h}^{n+1}\right]\right)$

$+b^{*}\left(2 \mathbf{u}_{h}^{n}-\mathbf{u}_{h}^{n-1}, E\left[\eta_{T}^{n+1}\right], E\left[\psi_{T, h}^{n+1}\right]\right)+\Lambda_{2}\left(\mathbf{u}, T, E\left[\psi_{T, h}^{n+1}\right]\right)$,

and

$\frac{1}{4 \Delta t}\left(\left\|\psi_{w, h}^{n+1}\right\|^{2}-\left\|\psi_{w, h}^{n}\right\|^{2}\right)+\frac{1}{4 \Delta t}\left(\left\|2 \psi_{w, h}^{n+1}-\psi_{w, h}^{n}\right\|^{2}-\left\|2 \psi_{w, h}^{n}-\psi_{w, h}^{n-1}\right\|^{2}\right)$

$+\frac{1}{4 \Delta t}\left(\left\|\psi_{w, h}^{n+1}-\psi_{w, h}^{n}\right\|^{2}-\left\|\psi_{w, h}^{n}-\psi_{w, h}^{n-1}\right\|^{2}\right)+\frac{3}{4 \Delta t}\left\|\psi_{w, h}^{n+1}-2 \psi_{w, h}^{n}+\psi_{w, h}^{n-1}\right\|^{2}$

$+v\left\|\nabla E\left[\psi_{w, h}^{n+1}\right]\right\|^{2}$

$=v\left(\nabla E\left[\eta_{w}^{n+1}\right], \nabla E\left[\psi_{w, h}^{n+1}\right]\right)+b^{*}\left(2 \mathbf{e}_{\mathbf{u}}^{n}-\mathbf{e}_{\mathbf{u}}^{n-1}, E\left[w^{n+1}\right], E\left[\psi_{w, h}^{n+1}\right]\right)$

$+b^{*}\left(2 \mathbf{u}_{h}^{n}-\mathbf{u}_{h}^{n-1}, E\left[\eta_{w}^{n+1}\right], E\left[\psi_{w, h}^{n+1}\right]\right)-R i\left(\frac{\partial\left(2 e_{T}^{n}-e_{T}^{n-1}\right)}{\partial x}, E\left[\psi_{w, h}^{n+1}\right]\right)$

$+\Lambda_{3}\left(\mathbf{u}, T, w, E\left[\psi_{w, h}^{n+1}\right]\right)$

We now bound the right hand side terms of (24). Apply the Cauchy-Schwarz inequality, Lemma 3, Lemma 4 to the first fourth right hand side terms which yields

$$
\begin{gathered}
\left|v\left(\nabla E\left[\boldsymbol{\eta}_{\mathbf{u}}^{n+1}\right], \nabla E\left[\boldsymbol{\Psi}_{\mathbf{u}, h}^{n+1}\right]\right)\right| \leq \frac{\varepsilon_{1} v}{2}\left\|\nabla E\left[\boldsymbol{\eta}_{\mathbf{u}}^{n+1}\right]\right\|^{2}+\frac{v}{2 \varepsilon_{1}}\left\|\nabla E\left[\boldsymbol{\Psi}_{\mathbf{u}, h}^{n+1}\right]\right\|^{2}, \\
\left|\left(P^{n+1}-r_{h}, \nabla \cdot E\left[\boldsymbol{\Psi}_{\mathbf{u}, h}^{n+1}\right]\right)\right| \leq \frac{\varepsilon_{2} v^{-1}}{2} \inf _{r_{h} \in Q_{h}}\left\|P^{n+1}-r_{h}\right\|^{2}+\frac{v}{2 \varepsilon_{2}}\left\|\nabla E\left[\boldsymbol{\Psi}_{\mathbf{u}, h}^{n+1}\right]\right\|^{2}, \\
\left|R i\left(\left(2 e_{T}^{n}-e_{T}^{n-1}\right) \widehat{\boldsymbol{k}}, E\left[\boldsymbol{\Psi}_{\mathbf{u}, h}^{n+1}\right]\right)\right| \leq R i\left(\left\|2 \eta_{T}^{n}-\eta_{T}^{n-1}\right\|+\left\|2 \psi_{T, h}^{n}-\psi_{T, h}^{n-1}\right\|\right) C_{P}\left\|\nabla E\left[\boldsymbol{\Psi}_{\mathbf{u}, h}^{n+1}\right]\right\|, \\
\leq \frac{R i^{2} C_{P}^{2} v^{-1} \varepsilon_{3}}{2}\left(\left\|2 \eta_{T}^{n}-\eta_{T}^{n-1}\right\|^{2}+\left\|2 \psi_{T, h}^{n}-\psi_{T, h}^{n-1}\right\|^{2}\right)+\frac{v}{\varepsilon_{3}}\left\|\nabla E\left[\boldsymbol{\Psi}_{\mathbf{u}, h}^{n+1}\right]\right\|^{2} .
\end{gathered}
$$

To estimate the first non-linear term below, use the error decomposition, the Hölder and the Young's inequalities to get

$$
\begin{aligned}
& \left|\left(\left(2 e_{w}^{n}-e_{w}^{n-1}\right) \times E\left[\mathbf{u}^{n+1}\right], E\left[\boldsymbol{\Psi}_{\mathbf{u}, h}^{n+1}\right]\right)\right| \\
& \leq\left|\left(\left(2 \eta_{w}^{n}-\eta_{w}^{n-1}\right) \times E\left[\mathbf{u}^{n+1}\right], E\left[\boldsymbol{\psi}_{\mathbf{u}, h}^{n+1}\right]\right)\right|+\left|\left(\left(2 \psi_{w, h}^{n}-\psi_{w, h}^{n-1}\right) \times E\left[\mathbf{u}^{n+1}\right], E\left[\boldsymbol{\Psi}_{\mathbf{u}, h}^{n+1}\right]\right)\right| \\
& \leq C\left(\left\|2 \eta_{w}^{n}-\eta_{w}^{n-1}\right\|+\left\|2 \psi_{w, h}^{n}-\psi_{w, h}^{n-1}\right\|\right)\left\|\nabla E\left[\mathbf{u}^{n+1}\right]\right\|\left\|\nabla E\left[\boldsymbol{\Psi}_{\mathbf{u}, h}^{n+1}\right]\right\|
\end{aligned}
$$


$\leq \frac{C \varepsilon_{4} v^{-1}}{2}\left(\left\|2 \eta_{w}^{n}-\eta_{w}^{n-1}\right\|^{2}+\left\|2 \psi_{w, h}^{n}-\psi_{w, h}^{n-1}\right\|^{2}\right)\left\|\nabla E\left[\mathbf{u}^{n+1}\right]\right\|^{2}+\frac{v}{\varepsilon_{4}}\left\|\nabla E\left[\boldsymbol{\Psi}_{\mathbf{u}, h}^{n+1}\right]\right\|^{2}$

The second nonlinear term is estimated as follows:

$$
\begin{aligned}
\left|\left(\left(2 w_{h}^{n}-w_{h}^{n-1}\right) \times E\left[\boldsymbol{\eta}_{\mathbf{u}}^{n+1}\right], E\left[\boldsymbol{\Psi}_{\mathbf{u}, h}^{n+1}\right]\right)\right| & \leq C\left\|2 w_{h}^{n}-w_{h}^{n-1}\right\|\left\|\nabla E\left[\boldsymbol{\eta}_{\mathbf{u}}^{n+1}\right]\right\|\left\|\nabla E\left[\boldsymbol{\Psi}_{\mathbf{u}, h}^{n+1}\right]\right\| \\
& \leq \frac{C v^{-1} \varepsilon_{5}}{2}\left\|2 w_{h}^{n}-w_{h}^{n-1}\right\|^{2}\left\|\nabla E\left[\boldsymbol{\eta}_{\mathbf{u}}^{n+1}\right]\right\|^{2}+\frac{v}{2 \varepsilon_{5}}\left\|\nabla E\left[\boldsymbol{\Psi}_{\mathbf{u}, h}^{n+1}\right]\right\|^{2} .
\end{aligned}
$$

Insert all these estimates to (24), and choose appropriate $\varepsilon_{i}, i=1,2, \ldots, 5$. Multiplying the resulting inequality by $4 \Delta t$ yields

$$
\begin{gathered}
\left(\left\|\boldsymbol{\Psi}_{\mathbf{u}, h}^{n+1}\right\|^{2}-\left\|\boldsymbol{\Psi}_{\mathbf{u}, h}^{n}\right\|^{2}\right)+\left(\left\|2 \boldsymbol{\Psi}_{\mathbf{u}, h}^{n+1}-\boldsymbol{\Psi}_{\mathbf{u}, h}^{n}\right\|^{2}-\left\|2 \boldsymbol{\Psi}_{\mathbf{u}, h}^{n}-\boldsymbol{\Psi}_{\mathbf{u}, h}^{n-1}\right\|^{2}\right) \\
+\left(\left\|\boldsymbol{\Psi}_{\mathbf{u}, h}^{n+1}-\boldsymbol{\Psi}_{\mathbf{u}, h}^{n}\right\|^{2}-\left\|\boldsymbol{\Psi}_{\mathbf{u}, h}^{n}-\boldsymbol{\Psi}_{\mathbf{u}, h}^{n-1}\right\|^{2}\right)+3\left\|\boldsymbol{\Psi}_{\mathbf{u}, h}^{n+1}-2 \boldsymbol{\Psi}_{\mathbf{u}, h}^{n}+\boldsymbol{\Psi}_{\mathbf{u}, h}^{n-1}\right\|^{2} \\
+2 v \Delta t\left\|\nabla E\left[\boldsymbol{\Psi}_{\mathbf{u}, h}^{n+1}\right]\right\|^{2}
\end{gathered}
$$$$
\leq C \Delta t\left(v\left\|\nabla E\left[\eta_{\mathbf{u}}^{n+1}\right]\right\|^{2}+v^{-1} \inf _{r_{h} \in Q_{h}}\left\|P^{n+1}-r_{h}\right\|^{2}+v^{-1} R i^{2} C_{P}^{2}\left\|2 \eta_{T}^{n}-\eta_{T}^{n-1}\right\|^{2}\right.
$$$$
+v^{-1} R i^{2} C_{P}^{2}\left\|2 \psi_{T, h}^{n}-\psi_{T, h}^{n-1}\right\|^{2}+v^{-1}\left\|2 \eta_{w}^{n}-\eta_{w}^{n-1}\right\|^{2}\left\|\nabla E\left[\mathbf{u}^{n+1}\right]\right\|^{2}
$$$$
\left.+v^{-1}\left\|2 \psi_{w, h}^{n}-\psi_{w, h}^{n-1}\right\|^{2}\left\|\nabla E\left[\mathbf{u}^{n+1}\right]\right\|^{2}+v^{-1}\left\|2 w_{h}^{n}-w_{h}^{n-1}\right\|^{2}\left\|\nabla E\left[\boldsymbol{\eta}_{\mathbf{u}}^{n+1}\right]\right\|^{2}\right)
$$$$
+4 \Delta t \Lambda_{1}\left(\mathbf{u}, T, w, E\left[\boldsymbol{\Psi}_{\mathbf{u}, h}^{n+1}\right]\right) \text {. }
$$

Now take $\mathbf{v}_{h}=E\left[\boldsymbol{\psi}_{\mathbf{u}, h}^{n+1}\right]$ in Lemma 8 , and choose $\varepsilon_{1}^{*}+\varepsilon_{2}^{*}+\varepsilon_{3}^{*}+\varepsilon_{4}^{*}=1 / 4$. Summing over time steps, and applying (20) for true solutions yields

$$
\begin{gathered}
\left\|\boldsymbol{\Psi}_{\mathbf{u}, h}^{N}\right\|^{2}+\left\|2 \boldsymbol{\Psi}_{\mathbf{u}, h}^{N}-\boldsymbol{\Psi}_{\mathbf{u}, h}^{N-1}\right\|^{2}+\left\|\boldsymbol{\Psi}_{\mathbf{u}, h}^{N}-\boldsymbol{\Psi}_{\mathbf{u}, h}^{N-1}\right\|^{2}+3 \sum_{n=0}^{N-1}\left\|\boldsymbol{\Psi}_{\mathbf{u}, h}^{n+1}-2 \boldsymbol{\Psi}_{\mathbf{u}, h}^{n}+\boldsymbol{\Psi}_{\mathbf{u}, h}^{n-1}\right\|^{2} \\
+v \Delta t \sum_{n=0}^{N-1}\left\|\nabla E\left[\boldsymbol{\Psi}_{\mathbf{u}, h}^{n+1}\right]\right\|^{2} \\
\leq C\left(v h^{2 k+2}\||\mathbf{u}|\|_{2, k+2}^{2}+v^{-1} h^{2 k}\left\|\left|P\left\|_{2, k}^{2}+R i^{2} C_{P}^{2} v^{-1} h^{2 k+2}\right\|\right| T \mid\right\|_{2, k+1}^{2}\right. \\
\left.+v^{-1} h^{2 k+2}\||\mathbf{u}|\|_{2, k+2}^{2}\|\mid \nabla \mathbf{u}\|_{\infty, 0}^{2}\right)+C v^{-1} \Delta t \sum_{n=0}^{N-1}\left\|2 w_{h}^{n}-w_{h}^{n-1}\right\|^{2}\left\|\nabla E\left[\boldsymbol{\eta}_{\mathbf{u}}^{n+1}\right]\right\|^{2} \\
+C \Delta t \sum_{n=0}^{N-1}\left(v^{-1} R i^{2} C_{P}^{2}\left\|2 \psi_{T, h}^{n}-\psi_{T, h}^{n-1}\right\|^{2}+v^{-1}\left\|2 \psi_{w, h}^{n}-\psi_{w, h}^{n-1}\right\|^{2}\left\|\nabla E\left[\mathbf{u}^{n+1}\right]\right\|^{2}\right)+C \Delta t^{4} .
\end{gathered}
$$

Similar estimates produce the following bounds for the temperature/vorticity errors:

$$
\begin{aligned}
& \frac{1}{4 \Delta t}\left(\left\|\psi_{T, h}^{n+1}\right\|^{2}-\left\|\psi_{T, h}^{n}\right\|^{2}\right)+\frac{1}{4 \Delta t}\left(\left\|2 \psi_{T, h}^{n+1}-\psi_{T, h}^{n}\right\|^{2}-\left\|2 \psi_{T, h}^{n}-\psi_{T, h}^{n-1}\right\|^{2}\right) \\
& +\frac{1}{4 \Delta t}\left(\left\|\psi_{T, h}^{n+1}-\psi_{T, h}^{n}\right\|^{2}-\left\|\psi_{T, h}^{n}-\psi_{T, h}^{n-1}\right\|^{2}\right)+\frac{3}{4 \Delta t}\left\|\psi_{T, h}^{n+1}-2 \psi_{T, h}^{n}+\psi_{T, h}^{n-1}\right\|^{2} \\
& \quad+\frac{\kappa}{2}\left\|\nabla E\left[\psi_{T, h}^{n+1}\right]\right\|^{2} \\
& \leq C\left(\kappa\left\|\nabla E\left[\eta_{T}^{n+1}\right]\right\|^{2}+\kappa^{-1}\left(\left\|2 \eta_{\mathbf{u}}^{n}-\eta_{\mathbf{u}}^{n-1}\right\|^{2}+\left\|2 \boldsymbol{\psi}_{\mathbf{u}, h}^{n}-\boldsymbol{\psi}_{\mathbf{u}, h}^{n-1}\right\|^{2}\right)\left\|E\left[T^{n+1}\right]\right\|_{2}^{2}\right. \\
& \quad+\kappa^{-1}\left\|\nabla\left(2 \mathbf{u}_{h}^{n}-\mathbf{u}_{h}^{n-1}\right)\right\|^{2}\left\|\nabla E\left[\eta_{T}^{n+1}\right]\right\|^{2}+\left|\Lambda_{2}\left(\mathbf{u}, T, E\left[\psi_{T, h}^{n+1}\right]\right)\right|,
\end{aligned}
$$

and 


$$
\begin{aligned}
& \frac{1}{4 \Delta t}\left(\left\|\psi_{w, h}^{n+1}\right\|^{2}-\left\|\psi_{w, h}^{n}\right\|^{2}\right)+\frac{1}{4 \Delta t}\left(\left\|2 \psi_{w, h}^{n+1}-\psi_{w, h}^{n}\right\|^{2}-\left\|2 \psi_{w, h}^{n}-\psi_{w, h}^{n-1}\right\|^{2}\right) \\
& +\frac{1}{4 \Delta t}\left(\left\|\psi_{w, h}^{n+1}-\psi_{w, h}^{n}\right\|^{2}-\left\|\psi_{w, h}^{n}-\psi_{w, h}^{n-1}\right\|^{2}\right)+\frac{3}{4 \Delta t}\left\|\psi_{w, h}^{n+1}-2 \psi_{w, h}^{n}+\psi_{w, h}^{n-1}\right\|^{2} \\
& +\frac{v}{2}\left\|\nabla E\left[\psi_{w, h}^{n+1}\right]\right\|^{2} \\
& \leq C\left(\left.v\left\|\nabla E\left[\eta_{w}^{n+1}\right]\right\|\right|^{2}+v^{-1}\left(\left\|2 \eta_{\mathbf{u}}^{n}-\eta_{\mathbf{u}}^{n-1}\right\|^{2}+\left\|2 \boldsymbol{\psi}_{\mathbf{u}, h}^{n}-\boldsymbol{\psi}_{\mathbf{u}, h}^{n-1}\right\|^{2}\right)\left\|E\left[w^{n+1}\right]\right\|_{2}^{2}\right) \\
& +v^{-1}\left\|\nabla\left(2 \mathbf{u}_{h}^{n}-\mathbf{u}_{h}^{n-1}\right)\right\|^{2}\left\|\nabla E\left[\eta_{w}^{n+1}\right]\right\|^{2}+v^{-1} R i^{2}\left(\left\|2 \eta_{T}^{n}-\eta_{T}^{n-1}\right\|^{2}\right. \\
& \left.+\left\|2 \psi_{T, h}^{n}-\psi_{T, h}^{n-1}\right\|^{2}\right)+\left|\Lambda_{3}\left(\mathbf{u}, T, w, E\left[\psi_{w, h}^{n+1}\right]\right)\right| .
\end{aligned}
$$

Now, set $\chi_{h}=E\left[\psi_{T, h}^{n+1}\right]$ and $s_{h}=E\left[\psi_{w, h}^{n+1}\right]$ in Lemma 8 , multiply by $4 \Delta \operatorname{tand}$ sum from $n=0$ to $N-$ 1 , and apply (20) to get

$$
\begin{gathered}
\left\|\psi_{T, h}^{N}\right\|^{2}+\left\|2 \psi_{T, h}^{N}-\psi_{T, h}^{N-1}\right\|^{2}+\left\|\psi_{T, h}^{N}-\psi_{T, h}^{N-1}\right\|^{2}+\left\|\psi_{T, h}^{N}-2 \psi_{T, h}^{N-1}+\psi_{T, h}^{N-2}\right\|^{2} \\
+\kappa \Delta t \sum_{n=0}^{N-1}\left\|\nabla E\left[\psi_{T, h}^{n+1}\right]\right\|^{2} \\
\leq C\left(\kappa h^{2 k}\||T|\|_{2, k+1}^{2}+\kappa^{-1} h^{2 k+4}\||\mathbf{u}|\|_{2, k+2}^{2}\||T|\|_{\infty, 2}^{2}\right) \\
+C \Delta t \sum_{n=0}^{N-1} \kappa^{-1}\left(\left\|\nabla\left(2 \mathbf{u}_{h}^{n}-\mathbf{u}_{h}^{n-1}\right)\right\|^{2}\left\|\nabla E\left[\eta_{T}^{n+1}\right]\right\|^{2}+\left\|E\left[T^{n+1}\right]\right\|_{2}^{2}\left\|2 \boldsymbol{\Psi}_{\mathbf{u}, h}^{n}-\boldsymbol{\Psi}_{\mathbf{u}, h}^{n-1}\right\|^{2}\right)+C \Delta t^{4},
\end{gathered}
$$

and

$$
\begin{aligned}
& \left\|\psi_{w, h}^{N}\right\|^{2}+\left\|2 \psi_{w, h}^{N}-\psi_{w, h}^{N-1}\right\|^{2}+\left\|\psi_{w, h}^{N}-\psi_{w, h}^{N-1}\right\|^{2}+\left\|\psi_{w, h}^{N}-2 \psi_{w, h}^{N-1}+\psi_{w, h}^{N-2}\right\|^{2} \\
& \quad+v \Delta t \sum_{n=0}^{N-1}\left\|\nabla E\left[\psi_{w, h}^{n+1}\right]\right\|^{2} \\
& \leq C\left(v h^{2 k}\||\mathbf{u}|\|_{2, k+2}^{2}+v^{-1} h^{2 k+4}\||\mathbf{u}|\|_{2, k+2}^{2}\||w|\|_{\infty, 2}^{2}+v^{-1} R i^{2} h^{2 \mathrm{k}+2}\||T|\|_{2, k+1}^{2}\right) \\
& +C \Delta t \sum_{n=0}^{N-1} v^{-1}\left(\left\|\nabla\left(2 \mathbf{u}_{h}^{n}-\mathbf{u}_{h}^{n-1}\right)\right\|^{2}\left\|\nabla E\left[\eta_{w}^{n+1}\right]\right\|^{2}\right. \\
& +C \Delta t \sum_{n=0}^{N-1} v^{-1}\left(\left\|2 \boldsymbol{\Psi}_{\mathbf{u}, h}^{n}-\boldsymbol{\psi}_{\mathbf{u}, h}^{n-1}\right\|^{2}\left\|E\left[w^{n+1}\right]\right\|_{2}^{2}+\left\|2 \psi_{T, h}^{n}-\psi_{T, h}^{n-1}\right\|^{2}\right)+C \Delta t^{4} .
\end{aligned}
$$

Summing the last two inequalities together with (27), and applying the Gronwall Lemma together with the velocity/temperature/vorticity stability results finishes the proof.

\subsection{Numerical experiment 1: convergence rate verification}

The first numerical experiment confirms the spatial and temporal convergence rates of Algorithm 1. Choosing $\boldsymbol{P}_{2}$ for the velocity, $P_{1}$ for the pressure, and $P_{2}$ for the vorticity/temperature, Theorem 9 concludes that spatial and temporal errors are both of second-order,i.e.,

$$
\begin{aligned}
& \left\|\mathbf{u}\left(t^{*}\right)-\mathbf{u}_{h}^{N}\right\|+\left\|\left|\mathbf{u}-\mathbf{u}_{h}\right|\right\|_{2,1}+\left\|T\left(t^{*}\right)-T_{h}^{N}\right\|+\left\|\left|T-T_{h}\right|\right\|_{2,1}+\left\|w\left(t^{*}\right)-w_{h}^{N}\right\|+\left\|\left|w-w_{h}\right|\right\|_{2,1} \\
& =O\left(h^{2}+\Delta t^{2}\right)
\end{aligned}
$$

where 


$$
\|\cdot\|_{2,1}:=\Delta \mathrm{t}\left(\sum_{\mathrm{n}=0}^{\mathrm{N}-1}\|\nabla(\cdot)\|_{2,1}^{2}\right)^{1 / 2}
$$

To verify these rates, the analytical solutions are chosen as

$$
\begin{gathered}
\mathbf{u}(\mathbf{x}, t):=\left\langle e^{t} \cos (\pi(y-t)), e^{t} \sin (\pi(x+t))\right\rangle, p(x, t):=\sin (x+y)\left(1+t^{2}\right) \\
T(x, t):=\sin (\pi x)+y e^{t}
\end{gathered}
$$

in $\boldsymbol{\Omega}:=(0,1) \times(0,1)$ with dimensionless flow parameters $v=1, R i=1, \kappa=1$. From which initial conditions and forcing terms are defined. To observe second order temporal accuracy, we first fix $h=$ $1 / 128$, and then calculate approximations for $\Delta t=\frac{1}{4}, \frac{1}{8}, \frac{1}{16}, \frac{1}{32}, \frac{1}{64}$. The results are presented in Table 1. In a similar manner, after setting $t^{*}=0.001, \Delta t=0.0001$, we run Algorithm 1 for $h=\frac{1}{4}, \frac{1}{8}, \frac{1}{16}, \frac{1}{32}$, $\frac{1}{64}$, see Table 2 . Both two results coincide with (28).

Table 1. Temporal errors and rates for a fixed mesh size $h=1 / 128$

\begin{tabular}{|l|l|l|l|l|l|l|}
\hline$\Delta t$ & $\left\|\left|\mathbf{u}-\mathbf{u}_{h}\right|\right\|_{2,1}$ & Rate & $\left\|\left|T-T_{h}\right|\right\|_{2,1}$ & Rate & $\left\|\left|T-T_{h}\right|\right\|_{2,1}$ & Rate \\
\hline $1 / 4$ & $5.3861 \mathrm{e}-1$ & --- & $4.4585 \mathrm{e}-1$ & --- & 2.4682 & --- \\
\hline $1 / 8$ & $1.5552 \mathrm{e}-1$ & 1.85 & $1.3711 \mathrm{e}-1$ & 1.70 & $6.5782 \mathrm{e}-1$ & 1.91 \\
\hline $1 / 16$ & $3.4477 \mathrm{e}-2$ & 2.22 & $3.5219 \mathrm{e}-2$ & 1.97 & $1.3904 \mathrm{e}-1$ & 2.24 \\
\hline $1 / 32$ & $8.0471 \mathrm{e}-3$ & 2.15 & $8.8744 \mathrm{e}-3$ & 1.99 & $3.1829 \mathrm{e}-2$ & 2.13 \\
\hline $1 / 64$ & $1.9507 \mathrm{e}-3$ & 2.09 & $2.2280 \mathrm{e}-3$ & 1.99 & $7.6539 \mathrm{e}-3$ & 2.06 \\
\hline
\end{tabular}

Table 2. Spatial errors and rates for an end time $t^{*}=0.001$ and $\Delta t=0,0001$

\begin{tabular}{|l|l|l|l|l|l|l|}
\hline$h$ & $\left\|\left|\mathbf{u}-\mathbf{u}_{h}\right|\right\|_{2,1}$ & Rate & $\left\|\left|T-T_{h}\right|\right\|_{2,1}$ & Rate & $\left\|\left|T-T_{h}\right|\right\|_{2,1}$ & Rate \\
\hline $1 / 2$ & $8.3533 \mathrm{e}-3$ & --- & $5.8896 \mathrm{e}-3$ & --- & $2.6225 \mathrm{e}-2$ & --- \\
\hline $1 / 4$ & $2.1083 \mathrm{e}-3$ & 1.99 & $1.4828 \mathrm{e}-3$ & 1.99 & $6.6020 \mathrm{e}-3$ & 1.99 \\
\hline $1 / 8$ & $5.1801 \mathrm{e}-4$ & 2.03 & $3.6277 \mathrm{e}-4$ & 2.04 & $1.6133 \mathrm{e}-3$ & 2.03 \\
\hline $1 / 16$ & $1.2761 \mathrm{e}-4$ & 2.02 & $8.9073 \mathrm{e}-5$ & 2.03 & $3.9599 \mathrm{e}-4$ & 2.03 \\
\hline $1 / 32$ & $3.1745 \mathrm{e}-5$ & 2.01 & $2.2134 \mathrm{e}-5$ & 2.01 & $9.8396 \mathrm{e}-5$ & 2.01 \\
\hline $1 / 64$ & $7.9238 \mathrm{e}-6$ & 2.00 & $5.5241 \mathrm{e}-6$ & 2.00 & $2.4576 \mathrm{e}-5$ & 2.00 \\
\hline
\end{tabular}

We point out that both $w_{h}$ and $\mathbf{u}_{h}$ approximate the same fluid variable, $\mathbf{u}$. Therefore, $\left\|w_{h}-\left(\nabla \times \mathbf{u}_{h}\right)\right\|$ should be very small. To observe this, we make three calculations when for $t^{*}=\frac{1}{16}, h=\frac{1}{32} ; t^{*}=$ $\frac{1}{32}, h=\frac{1}{64}$ and $t^{*}=\frac{1}{64}, h=\frac{1}{128}$. We labelled the plots obtained from these calculations by Level 1 , Level 2 and Level 3, respectively. The results can be seen in Figure 1. 


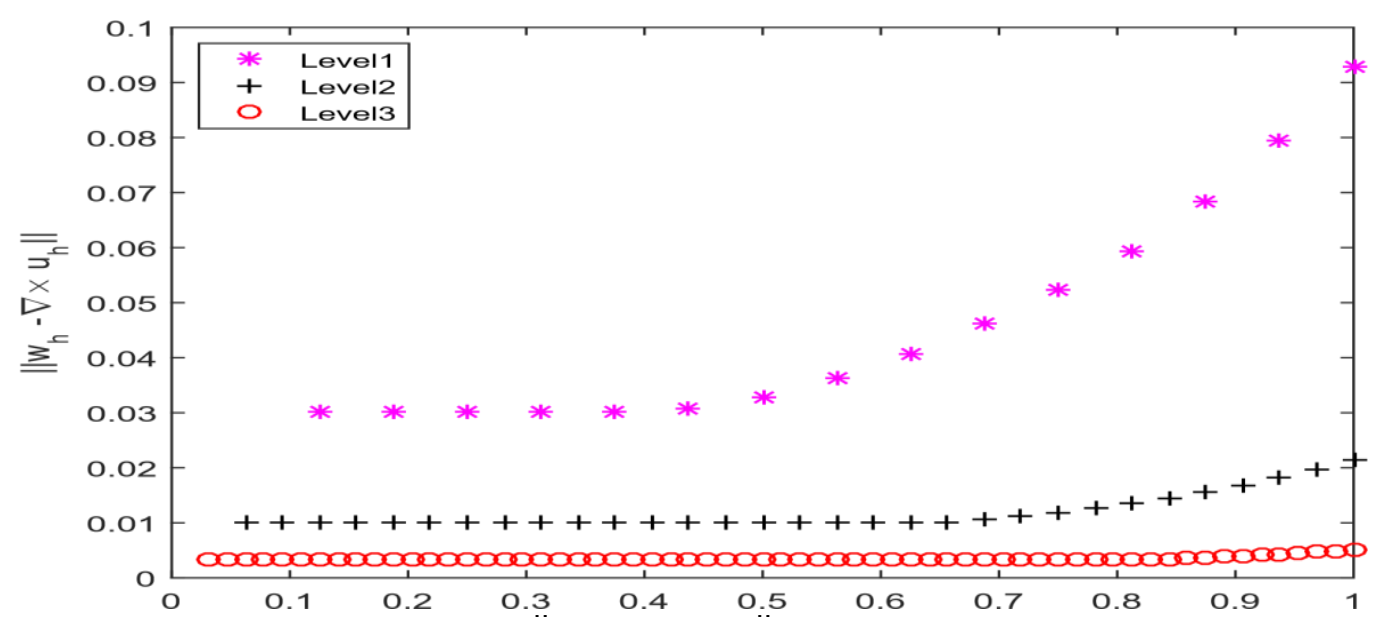

Figure 1. Shown above is a plot of $\left\|w_{h}-\nabla \times \mathbf{u}_{h}\right\|$ versus time, for the finest 3 discretizations

\subsection{Numerical experiment 2: Marsigli's experiment with $R e=1000$}

The second numerical experiment tests the proposed algorithm on a benchmark problem, named Marsigli's experiment. For the problem set-up, we follow the paper [18]. Flow region is an insulated box $[0,8] \times[0,1]$ divided at $x=4$. The initial velocity is taken to be zero since the flow is at rest, and the initial temperature on the left hand side of the box is $t^{0}=1.0$, and on the right hand side $t^{0}=1.5$. The dimensionless flow parameters are set to be $R e=1000, R i=4, \operatorname{Pr}=1$ and the flow starts from rest.

Our goal here is to compare Algorithm 1 and BE-FEM of the VVT-model on the same moderately fine mesh. Taking the same flow parameters as in DNS except time step $\Delta t=0.02$, we calculated all solutions at $t^{*}=2,4,6,8$ on the same mesh. The results from these computations are shown in Figure 3. Even though BE-FEM gives very similar results at $t^{*}=2, t^{*}=4$ to those of the paper [18], see Figure 2, it produces time delay solutions $t^{*}=6$ and $t^{*}=8$. However, Algorithm 1 catches very well the flow pattern and temperature distribution as in [18] at all time levels, $t^{*}=2,4,6$, 8. Hence, the proposed method dramatically improves the results of BE-FEM for VVT model.
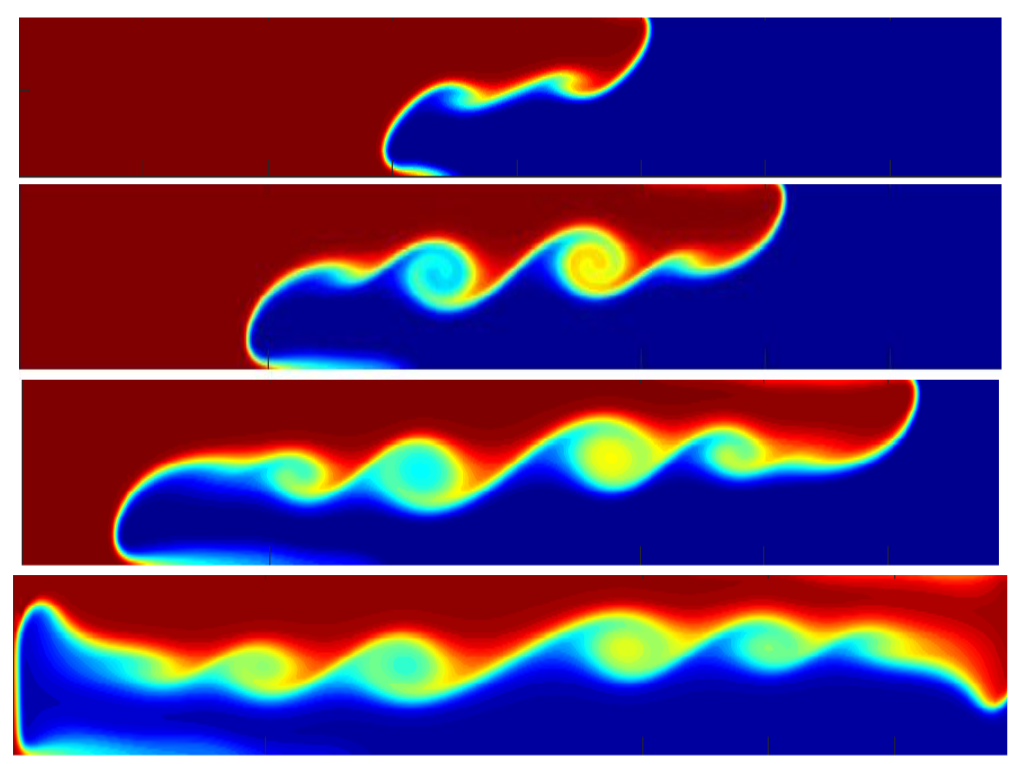

Figure 2. The temperature contours of direct numerical simulaitons for Boussinesq equations, respectivelty, at $t^{*}=2,4,6,8$ 

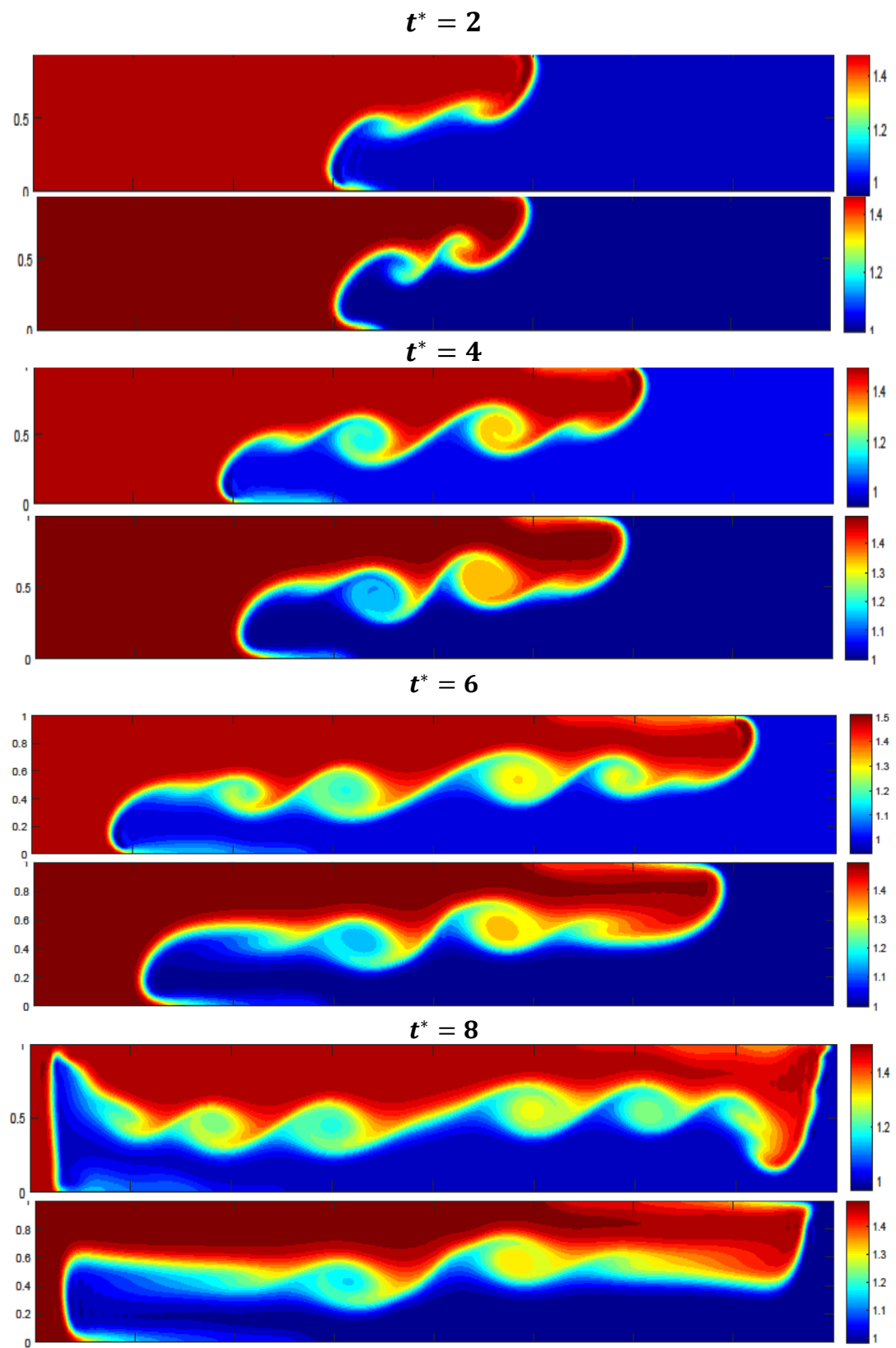

Figure 3. The temperature contours of our model and of BE-FEM for VVT-model, respectively, at $t^{*}=2,4,6,8$

\section{CONCLUSIONS}

In this paper, we proposed, analyzed and tested a numerical method for VVT model of the Boussinesq equations. The method combines the standard BE-FEM with three modular second order linear time filters, one each for the velocity, the temperature and the vorticity. Since these filter steps are implemented as completely decoupled steps, the method requires only three lines of code into the standard BE-FE code at each time step. Eliminating intermediate steps leads to an equivalent method which is second order, A-stable. It was proven that approximate solutions are unconditionally stable in time, and possess optimal convergence rates. The numerical experiments presented here verify theoretical convergence rates, and reveal the superiority of the method over the standard BE-FEM of the VVT model on a benchmark problem, named Marsigli's experiment. 


\section{CONFLICTS OF INTEREST}

No conflict of interest was declared by the author.

\section{REFERENCES}

[1] John, V., Matthies, G., Rang, J. A., "Comparison of time-discretization/linearization approaches for the incompressible navier-stokes equations", Computer Methods in Applied Mechanics and Engineering., 195: 5995-6010, (2006).

[2] Frutos, J., Garcia-Archilla , B., Novo, J., "The post-processed mixed finite-element method for the navier-stokes equations: refined error bounds", SIAM Journal of Numerical Analysis, 46(1): 201-230, (2008).

[3] Gresho, P. M., Sani, R. L., "Incompressible flow and the finite element method, Volume 2, Isothermal Laminar Flow”, Wiley, (1998).

[4] Guzel, A., Layton, W., "Time filters increase accuracy of the fully implicit method", BIT Numerical Mathematics, 58(2): 301-315, (2018).

[5] Decaria, V., Layton, W., Zhao, H., "A time-accurate, adaptive discretization for fluid flow problems", International Journal of Numerical Analysis and Modeling, 17(2): 254-280, (2020).

[6] Cibık, A., Kaya, S., "A projection based stabilized finite element method for natural convection problem", Journal of Mathematical Analysis and Applications, 381(2): 469-484, (2011).

[7] Cengel, Y.A., Ghajar, A.J., Heat and Mass Transfer: Fundamentals and Applications 5th ed. , McGraw-Hill Education, New York, (2015).

[8] Wong, K. L., Baker, A. J., “A 3d incompressible navier-stokes velocity-vorticity weak form finite element algorithm", International Journal of Numerical Methods in Fluids, 38(2): 99$123,(2002)$.

[9] Wang, C., Liu, J. -G. "Analysis of finite difference schemes for unsteady navier-stokes equations in vorticity formulation", Numerical Mathematics, 91(3): 543-576, (2002).

[10] Lee, H. L., Olshanskii, M. A., Rebholz, L. G., "On error analysis for the 3d navier-stokes equations in velocity-vorticity-helicity form", SIAM Journal of Numerical Analysis, 49(2): 711-732, (2011).

[11] Olshanskii, M. A., "A fluid solver based on vorticity-helical density equations with application to a natural convection in a cubic cavity", International Journal of Numerical Methods in Fluids, 69(5): 983-994, (2012).

[12] Heister, T., Olshanskii, M.A., Rebholz, L. G., "Unconditional long-time stability of a velocityvorticity method for 2d navier-stokes equations", Numerical Mathematics, 135(1): 143-167, (2017).

[13] Akbas, M., Rebholz, L. G., Zerfas, C., "Optimal vorticity accuracy in an efficient velocityvorticity method for the $2 \mathrm{~d}$ Navier-Stokes equations", Calcolo, 55(3): 1-29, (2018). 
[14] Cibık, A., Demir M., Kaya S., "A family of second order time stepping methods for the DarcyBrinkman equations", Journal of Mathematical Anlaysis and Applications, 472(1): 148-175, (2020).

[15] Cibık, A., Eroğlu, F. G., Kaya, S.,"Analysis of Second Order Time Filtered Backward Euler Method for MHD Equations", Journal of Scientific Computing, 82(2): 2020.

[16] Adams, R., Fournier, J.F.,Sobolev Spaces 2 nd. ed., Academic Press, Newyork, 1975.

[17] Layton, W., Introduction to Finite Element Methods, SIAM, Philadelphia, (2008).

[18] Wang, C.,Liu, J. -G., Johnston, H., "Analysis of a fourth order finite difference method for the incompressible boussinesq equations", Numerical Mathematics, 97(3): 555-594, (2004). 